\title{
Experimental Investigation of Line-Tying Effects on the Spheromak Tilt Mode
}

\author{
F.J. Wysocki a) \\ Plasma Physics Laboratory, Princeton University, \\ Princeton, New Jersey 08544, USA
}

\begin{abstract}
A tariety of passive stabilization techniques compatible with $S-1$ inductive spheromak formation has been evaluated for eflectiveness against the MHD tilting instability. A line-tying stabilization effect has been previously identified theoretically. In the present work, the effects have been experimentally identified and investigated. The perturbed current patterns within passive stabilizing structures have been measured, and line-tying currents have been identified. The simple ring model of tilting has been expanded to include line-tying, and has proviled estimates for growth rates which agree well with the measured values.
\end{abstract}

a) Present address: AT\&T Bell Laboratories, Murray Hill, NJ 07974

PACS 52.30.Gz 52.35.Py 52.40.Hf 


\section{INTRODUCTION}

The inc reasing complexity and cost of experimental fusion devices (e.g., tokamaks and mirrors) have led to increased interest in alternate approaches, such as the reverse field pinch (RFP), field reversed configuration (FRC), and the spheromak. These devices may lead to smaller, cheaper fusion reactcrs.

One potential drawback of these devices is the possibility that nearby walls may be necessary for global stability. ${ }^{1-4}$ However, nearby walls are also being used in advanced tokamak designs, surh as PBX, ${ }^{5}$ for stabilizing axis; mmetric vertical instabilities. In addition, theoretical mirror studies are beginining to reevaluate the stabilizing effects provided by nearby conducting walls. ${ }^{6 \rightarrow 0}$ Thus, wall stabilization is not necessarily limited to RFP. FRC, and spheromak devices.

In the absence of close fitting walls or conducting elements, the spheromak is unstable to at least one global MHD mode (tilting or shifting).$^{10-12}$ Since these mades lead to destruction of the configuration, it is essential to have control over these instabilities to maintain the viability of the spheromak as a potential fusion ractor. Spheromals tilting and shifting have been the subject of recent theoretical ${ }^{4,13-18}$ and experimental ${ }^{19,20}$ investigation. Due to the difficulty of stabilizing these modes, several different stabilization techniques have been studied, including passive coils, ${ }^{19-2 I}$ line-tying, ${ }^{22-24}$ and theoretical calculations for gyroscopic $^{25}$ and finite-gysoradius-beam-particle ${ }^{26,27}$ stabilization. However, the techniques that are currently in use may not be suitable for reactor conditions. As spheromak research progresses toward reactor-relevant regimes, the question of optimal stabilization for global modes will probab!y remain.

Line-tying stabilization is theoretically shown to differ from purely inductive siabilization, ${ }^{24}$ which is identified by the typical "eddy" pattern satisfying the 
condition:

$$
\text { Pure induction: } \quad \vec{\nabla} \cdot \vec{J}_{\text {wall }}=0 \text {. }
$$

Line-tying effects are identified when the following condition occurs:

$$
\text { Line-tying : } \quad \vec{\nabla} \cdot \bar{J}_{\text {wall }} \neq 0,
$$

which implies current (referred to as line-tying current) enters (leaves) the wall from (to) the plasma.

An important criterion for a tilt (or shift) stabilizing scheme is that it be compatible with the spheromak formation technique. Several different techniques have successfully demonstrated spheromak formation. ${ }^{11,28-30}$ One technique. the $S-1$ inductive formation scheme, ${ }^{29}$ was first demonstrated in the Proto $S-$ $1 / A$ device, ${ }^{33}$ and later in Proto S-1/C (twice as big). ${ }^{32}$ and $S-1$ (six times the size of Proto S-1/A). ${ }^{33}$ The Proto S-1/C device was used in the experiments $\mathbf{r e}_{\mathrm{j}}$,orted here and is briefly described in Sec. II.

The Proto S-1 devices have provided a test bed for studying proposed tilt stabilization schemes (the shift mode is stable in these devices). Two passive stabilizing coil systems, the "figure 8 " and "saddle" coils, were previously developed $^{21}$ and found not to interfere with inductive formation. In an effort to extend the performance of passive stabilization in S-1 type devices. several other types of passive systems have been experimentally investigated in the Proto S1:C device. These systems include solid and spoked walls mounted above and below the spheromak and the "full enclosure" system, where the spoked walls are electrically connected. The results show that stabilization effects are small when the conductors are too far from the separatix surface. If the conductors are within the separatrix sur.ace, significant stabilization effects are found. In particular, line-tying effects are found to be important for nonoptimized stakilizers. Improvement in design (electrical connection of the spoked walls) 
substantially reduces the importance of line-tying. The resu!ts obtained with these passive systems are presented in Sec. III.

Previous theoretical ${ }^{22-24}$ and experimental ${ }^{20}$ work suggested that a linetying effect could play a role in slowing down the tilting motion. During the present investigation, the importance of line-tying effects became increasingly apparent. To investigate this effect further, the pert urbed current pattern within the passive coil structure was measured. Perturbed line-tying currents are easily identified in the "wagon wheel" (spoked wall) coil structure, as reported in Sec. IV.

Finally, an extension of the ring model of tilting, ${ }^{20}$ which includes line-tying stabilization in a simplified way, is presented in Sec $V$. This model is inherently different from previous theoretical calculations of line-tying stabilization, primarily in the treatment of the plasma-conducting wall boundary conditions. While previous calculations ${ }^{22-24}$ were in reasonable qualitative agreement with the experiments, the predicted strength of the line-tying effect appears to be somewhat optimistic when compared to the experimental growth rates. The model presented in this paper gives growth rate estimates which agree well with the measured values.

Concluding remarks are made in Sec. VI.

\section{EXPERIMENTAL APPARATUS}

The Proto S-1/C device (shown in Fig. 1) uses quasistatic $S-1$ induclive formation. ${ }^{29}$ The flux core has a $30-\mathrm{cm}$ major radius and a $6-\mathrm{cm}$ minor radius. and the stainless steel vacuum vessel is cylindrical with a radius of $75 \mathrm{~cm}$ and a height of $100 \mathrm{~cm}$. The flux core is powered by a $90 \mu \mathrm{F}-40 \mathrm{kV}$ capacitor bank used to produce the toroidal field (TF), and a $120 \mu \mathrm{F}-20 \mathrm{kV}$ bank for the poloidal field (PF). Typical operating voltages for the TF bank are $15-26 \mathrm{kV}$, 
and $6-13 \mathrm{kV}$ for the PF bank. The quarter period rise time is 65 and 70 as for the TF and PF circuits, respectively.

The required external equitibrium field is provided by a combination of steady state magnetic field coils outside the vacuum vessel and field produced by the flux core. The curvature index, $n_{i} \equiv\left(-r / B_{z}\right)\left(\partial B_{z} / \partial r\right)_{1}$ is dependent or the core currents, and can be adjusted by changing the crowbar time of the PF circuit. After the formation period, the index is typically $-0.5 \leqslant n_{i} \leqslant 0$, therefore the tilting mode is strongly unstable. With the existing atrangement, it is dificult to raise the index significantly above zero without adversely affecting the formation phase.

The internai magnetic field configuration is measured with a probe array consisting of many pickup loops. The array is capable of simultareous measurement of the three orthogonal components of $\vec{B}$ at five radial positions, spaced between the geometric symmetry axis and the edge of the fiux core. The array is movable in the $Z$ direction ( 1 to the midplane), and can be rotated to different toroidal angles. Using this mobility, multidischarge data are used to reconstruct the tiwe-dependent internal configuration. The success of this reconstruction is dependent on very good plasma reptoducibility, which is generally the case.

For the purpose of the line-tying study, knowledge of the plasma temperature $\left(T_{e}\right)$ and density $\left(n_{e}\right)$ are needed. The values for $T_{e}$ and $n_{e}$ were obrained from single point $(R=12 \mathrm{~cm}, Z=0$ ) Thomson scattering performed with a $6 \mathrm{~J}$ Q-swiched ruby laser and a 6-channel polychromator. The system layout was similar to that of a previously described system, ${ }^{34}$ except for the changes noted here. The signals from the polychromator were split and integrated with two (per channel) LeCroy 2249 gated integrators; one gated during the laser pulse. and one gated after the laser pulse. This allowed subtraction of the background plasma light. High background light levels caused low signal-to-noise ratios in 
the Thomson scattering data. Statistics usually prevented accurate determination of the temperature, or ciensity, in single discharges. The data reported in this paper are a result of averages of many discharges. No discrimination on individual discharges was employed; all discharges in a given sequence were included in the averages. Absolute calibration of Thomson scattering electron density was determined by Raman scattering. ${ }^{35}$ All of the experiments described in this paper were performed with a $10 \mathrm{~m}$ Torr fill pressure of hydrogen gas.

\section{PASSIVE STABILIZATION SYSTEMS}

Two passive coils systems, the "figure 8" and "saddle" coils, have previously been reported. ${ }^{19-21}$ These coils were designed to meet the constraint of absolutely no axisymmetric magnetic coupling with the flux core. Passive system design has been extended by allowing some axisymmetric coupling with the core, and the effects of this coupling have been evaluated, aloing with the overall tilt stabilizing effectiveness. These systems include solid and spoked walls mounted above and below the spheromak and the "full enclosure" system, where the spoked wails are electrically connected.

Results show that passive conductors with fairly large axisymmetric coupling $(\sim 30 \%)$ to the flux core do not prevent spheromak formation. However, induced currents in the conductors can alter the plasma shape considerably. which then affects the stabilization effectiveness of the condnctors. If the altered shape causes the cenductors to be outside the separatrix surface, then the stabilization effectiveness of those conductors is found to be very small. This alteration of shape can be controlled by appropriate design modifications, When the conductors are within the separatrix surface, significant stabilization effects are founc. In particular, line-tying effects are identified. Electrically connecting 
the spoked walls improves the performance of the stabilizers. The results which support these conclusions are presented in the following subsections.

\section{A. SOLID HORIZONTAL WALL}

The first such design is a pair of solid-conducting-horizontal walls placed above and below the spheromak. These walls consisted of disks $24 \mathrm{~cm}$ in radius (same as the inner edge of the flux core), positioned in $\mathrm{Z}$ at $\pm 11.5 \mathrm{~cm}$, with a 1.7-cm-radius center hole to allow probe access. Three values of wall resistance (ranging from very resistive to highly conductive) have been examined by changing the material type and thickness (see Table 1). Experimentally measured fux. contours for the three cases are shown in Fig. 2. Each plot corresponds to the time in the discharge when the tilted angle is 5 degreas, which is taken as the initiation of the tilt mode.

The presence of a perfectly conducting wall is expected to affect the inductive formation efficiency. An estimate of this effect is made by calculating the se!r-consistent current distribution induced within the wall by the poloidal field (PF) windings in the flux core. The total current induced in the wall is calculated to correspond to $37 \%$ of the PF current, which reduces the total vacuum poloidal fiux swing by $32 \%$. Fxperimentally, discharges with the highly conductive (copper) wall have a $29 \%$ reduction in poloidal flux as compared with discharges with the very resistive (stainless steel) wall (which had little effect on the total induced plasma $\mathrm{flux}$ ). This agreement may be fortuitous though, since there is strong axperimental evidence that Taylor relaxation mechanisms play an important role in determining the precise flux levels. ${ }^{33,36}$

Calculated resistive decay times of induced axisymmetric currents for the different walls are given in Table 1 . The decay time in the thin stainless steel wall is comparable to the $20 \mu$ s formation time scale, and thus, wall currents will 
have substantially decayed by the end of formation, and do not greatly affect the final equilibrium. Axisymmetric currents persist (at a somewhat decayed level) throughout the plasma lifetime in the thick stainless steel wall, and noticeably modify the equilibrium. The copper wall is perfectly conducting over the lifetime of the spheromak, and modifies the equilibrium very strongly.

Figure 2 shows that strong axisymmetric currents in the copper wall compress the equilibrium in the $Z$ direction. In this configuration, the wall is actually quite far from the separatrix surface (plasma edge). The ratio of the distance between the separatrix and the wall to the distance between the midpiane and the separatrix at $R=15.6 \mathrm{~cm}$ is 0.36 . Ideal MHD predicts this configuration to be unstable. ${ }^{15,37}$

If the axisymmetric currents decay (as in the resistive wall), the equilibrium expands in $Z$, and the separatrix approaches and finally passes through the wall (see Fig. 2). Not only is the wall comparatively closer to the plasma, but internal field lines now penetrate the wall. Tilting motion will tend to drag the lines through the wall, as shown in Fig. 3, which is possible because of finite wall resistance. This drag will slow down the tilting.

The measured time dependence of the tilt angle for the three cases of wall resistance are shown in Fig. 4. The growth rate is determined by a least squares fit of the data to $\ln \theta=\gamma\left(t-t_{0}\right)$. The measured growth rates (accurate to approximately $10-15 \%$ ) for the three cases are: $\gamma$ tbick $c u=0.28 \mu \mathrm{s}^{-1}$, $\gamma_{\text {thick s.s. }}=0.076 \mu \mathrm{s}^{-1}$, and $\gamma_{\text {thin s.s. }}=0.064 \mu \mathrm{s}^{-1}$.

As expected, the perfectly conducting wall produces an extremely tilt unstable spheromak, while the very resistive wall provides a sizeable stabilizing force, which reduces the growth rate by more than a factor of 4 . For comparison, the measured growth rate for discharges without any auxiliary stabilization is $0.33 \mu \mathrm{s}^{-1}$. 
The above discussion suggests that the stabilization effectiveness of the resistive wall is limited by its resistance. In fact, the measured growth time for the discharge with the $0.51-\mathrm{mm}$ stainless steel wall is very similar to the resistive decay time given in Table 1 . However, if the wall resistance is reduced, fewer internal field lines penetrate the wall, and the growth rate increases. The design modification described in the next subsection allows low resistance wall material to be used without undue compression in $\mathrm{Z}$, testing the idea that wall resistance limits the stabilization effectiveness.

\section{B. WAGON WHEEL}

To prevent strong compression in $\mathrm{Z}$, it is necessary to limit the toroidal currents in the wall. It is not sufficient to simply cut the wall along one radius, because the boundary condition on the wall, $\partial B_{\perp} \partial t=0$, is a local condition satisfied everywhere on the wall surface. The only effect of a radius cut would be a large perturbation on the plasma near the gap, which was experimentally confirmed.

The toroidal wall cursent would be eliminated by using a material with perfect conductivity in the radial direction, and zero conductivity in the toroidal direction. This idealized wall could be simulated by infinitely many radial cuts in a copper wall, or a "wall" comprised solely of a finite number of radial spokes. This is the motivation for the "wagon wheel" structure.

The "wagon wheel", constructed from 0.64-cm-diameter copper rod, con!sists of 10 radjal spokes (later increased to 20), an inrer hoop of radius $1.7 \mathrm{~cm}$. and an outer hoop with a $24-\mathrm{cm}$ radius. The wheels were positioned at $=11 . \overline{\mathrm{cm}}$ in Z. The inner hoop permits probe access. The outer hoop provides mechanical support for the rods and allows the possibility of "figure 8 " shaped perturbed current patterns. The axisymmetric current induced in the outer hoop provides 
additional external equilibrium field, which somewhat improves spherornak formation. ${ }^{38}$ Flux conservation within the hoops represents two global boundary conditions, as opposed to the infinity of local boundary conditions on a solid wall. Thus, the plasma assumes an equilibrium shape which is only slightly modified, and extreme compression in $Z$ does not occur (compare Figs, 2 and 6).

The current induced in the hoop by the PF windings in the flux core is calculated to be $23 \%$ of the PF current, which reduces the total vacuum poloidal flux swing by $26 \%$. Experimentally, discharges with the wagon wheel stabilizers have a $21 \%$ reduction in total poloidal flux compared to discharges : vith thin stainless steel walls.

The measured growth rate for discharges with wagon wheel stabilizers is $0.056 \mu \mathrm{s}^{-1}( \pm 10-15 \%)$, which is not significantly different from the case with $0.51-\mathrm{mm}$-thick stainless steel wall stabilizers.

The conductivity of copper is $\simeq 42$ times greater than stainless steel, and the cross-sectional area (for perturbed current flow) of the wagon wheel (with 10 spokes) is $\sim 65 \%$ of the solid $0.51-\mathrm{mm}$-thick wall. Thus, the wall resistance of the wagon wheel is $\sim 28$ times less than the stainless steel wall. The fact that the growth rate is roughly the same in both conditions indicates that the wall resistance is not limiting the effectiveness of this stabilizing technique. It is possible that the wagon wheel design has restricted the possible perturbed cursent patterns and this could reduce its intrinsic effectiveness. The results presented in Sec. IV indicate, however, that the wheel design does not rest rjct the desired current pattern more significantly than the solid wall.

\section{FULL ENCLOSURE}

Ideal MHD studies suggest that a fully enclosing wall will be more effective than horizontal walls alone. The calculated perturbed current pattern for a 
spherical enclosing wall ${ }^{15}$ is shown in Fig. 5. A sirnple 2 idition to the previous wagon wheel design allows a current pattein similar to that in Fig. 5 t,o flow, which should jmprove the performance of the stabilizer.

The perturbed current is allowed to flow from the top wheel to the bottom wheel by connecting them electrically with rod: (see Fig. 6). The connectors, made of 0.64-cm-diameter copper rod, are attached to the outer hoops of the wagon wheels and cross the midplane at a radius of $39 \mathrm{~cm}$, surrounding the flux core. The connectors do not couple magnetically to the flux core, and should not affect formation or equilibrium shape.

The number of radial spokes in the wagon wheel was increased from 10 to 20, and a comparison of performance for no connectors, 10 connectors, and 20 connectors was made (all conditions had 20 spokes). The data presented in this subsection represent discharges with $\sim \frac{1}{2}$ the toroidal plasma current of discharges in the previous subsections. This reduction of plasma current, due to flux core failure, makes direct comparison of results with 10 and 30 spokes difficult, because the data inciude current scaling effects. This will be discussed further in Sec. V-B.

Measured flux contours for the three cases $(0,10$, and 20 connectors) are shown in Fig. 6 at the $\theta=5^{\circ}$ time points. The measured growth r-tes (accirate to approximately $10-15 \%)$ for the three cases are: $\gamma$ a cann. $=0.083 \mu 5^{-1}$, $\gamma_{10}$ conn. $=0.045 \mu \mathrm{s}^{-1}$, and $\gamma_{20}$ conn. $=0.058 \mu \mathrm{s}^{-1}$. The $\theta=5^{\circ}$ time points for the three cases are: $t_{0} \operatorname{cosn} .=48 \mu \mathrm{s}, t_{j 0} \operatorname{conn} .=41 \mu \mathrm{s}$, and $t_{20} \operatorname{conn} .=61 \mu \mathrm{s}$ (all are $\pm 3 \mu \mathrm{s}$ ). The addition of 10 connectors reduced the growth rate by a factor of $\sim 2$, but tilting began earlier. Overall, the improvement is slight.

The largest improvement occurs in the 20 connector configuration. While the growth rate is comparable to the 10 connector case, there is a significant delay $(\approx 20 \mu \mathrm{s})$ in the onset of the tilting. This delay allows complete detachment of 
the spheromak from the $\mathrm{flux}$ core. In addition, the 20 connector case exhibits an oscillatory tilting behavior from $10 \mu \mathrm{s}$ to $60 \mu \mathrm{s}$, which is illustrated in Fig. 7 . This oscillatory motion ceases at $\sim 60 \mu \mathrm{s}$, a d simple exponential growth follows. The reproducibility of the phenomenon, even after complete reassembly of the enclosure, suggests that it is not simply experimental error.

A possible explanation for these oscillations can be obtained by examining the equations used by C. Munson et al. ${ }^{20}$ and C.C. Goldenbaum et al., ${ }^{39}$ which give

$$
\gamma^{2}=I_{x x}^{-1}\left[\frac{\pi}{c} R_{J}^{2} I_{p} B_{v}-I_{p}^{2} L_{c}^{-1} \frac{\partial M_{c p}}{\partial q}\left(\frac{\partial M_{c p}}{\partial \theta}+\frac{1}{6} \frac{\partial^{3} M_{c p}}{\partial \theta^{3}} \theta^{2}+\cdots\right)\right],
$$

where $\gamma$ is the tilting growth rate, $J_{x x}$ is the moment of inertia, $L_{c}$ is the inductance of the stabilizing coil, $M_{c p}$ is the mutual inductance between the stabilizing coil and the plasma, $I_{p}$ is the toroidal plasma current, $B_{u}$ is the externally applied "vertical" field, and $R_{J}$ is the major radius of the peak toroidal current. Oscillatory behavior cen occur when $y^{2}<0$.

In the Proto S-1 experiments, it is reasonably accurate to take $B_{v}$ as constant in time, $B_{v}=C_{v} I_{p_{0}} R_{J}^{-1}$, where $C_{v}$ is the appropriate constant of proportionality, and $I_{p 11}$ is the initial plasma current. Using this form of $B_{v}$, the condition for stable oscillations can be written as

$$
I_{p}>\frac{\pi}{c} R_{J} C_{v}\left(\frac{\partial M_{c p}}{\partial \theta}\right)^{-2} L_{\mathrm{c}} I_{p_{0}}
$$

In this case, oscillations may occur until the plasma current decays to the point where Eq. (1) is no longer satisfied.

However, when Eq. (1) is not satisfied, oscilla:ory behavior (most likely superposed on exponential growth) can occur if $\partial^{3} M_{c p} / \partial \theta^{3}$ is large enough. In this case, the value of $\gamma^{2}$ becomes negative for the following condition:

$$
\theta^{2}>6\left(\frac{\partial M_{c p}}{\partial \theta}\right)^{-\ddot{z}}\left(\frac{\partial^{3} M_{c p}}{\partial \theta^{3}}\right)^{-1}\left[\frac{\pi}{c} L_{c} R_{J} C_{v} J_{p_{0}} J_{p}^{-1}-\left(\frac{\partial M_{c p}}{\partial \theta}\right)^{2}\right] \text {. }
$$


The value of $\theta$ determined by Eq. (2) separates the concave-up and concavedown regions in the curve $\theta$ versus time.

The conditions given by Eqs. (1) and (2) become increasingly difficult to satisfy as the spheromak resistively decays and $I_{p}$ drops. In addition, the spheromak shrinks in major radius during the decay phase, since $B_{v}$ is roughly constant. Unfortunately, the coupling coefficient $M_{c p}$ decreases as the major radius decreases, adding to the difficulty in satisfying the constrajnts. If neither Eqs. (1) nor (2) are satisfied, then simple exponential growth is predicted. Thus, if oscillations occur at some point, eventually pure exponential growth will follow.

This discussion assumes the resistive current decay in the stabilizing coil can be ignored, which, for this experiment, is a very good approximation. Measurements of the coil resistance (including the coniact resistance due to the mechanical connections) and inductance show that the resistive decay time is $\approx 1.0 \mathrm{~ms}, 50$ times longer than the $20 \mu$ s time scale of the experimental $\gamma$ values.

Even though the addition of 20 connectors leads to improvement in performance, the spheromak eventually still suffers from a tilting mode. This is not surprising, since ideal MHD predicts fairly stringent requirements on the closeness of stabilizing conducting walls. ${ }^{4,15,1 ;}$ This may be expressed as the maximum allowable spatial gap between the separatrix and the wall, normalized to the poloidal minor radius. This value has been previously calculated to be $\simeq 0.2$ for an equilibrium similar to the experimental equilibrium. ${ }^{15}$ For the case in Fig. 6 (with 20 connectors), the gap (at the midplane) between the separatrix and the connectors is $\simeq 16.5 \mathrm{~cm}$ and the minor radius is $\simeq 13.3 \mathrm{~cm}$. Complete stability is not expected since this gap is greater than the rninor radius. In addition, the lack of total stability with 20 connectors is consistent with the experimental results from the CTX spheromak device, which has reasonably 
confirmed the MHD predictions for the CTX configuration. ${ }^{40}$ (The CTX group can control the gap fairly precisely by using an external dc bias field.) For a sufficiently small gap, the CTX group reports complete tilting stability, ${ }^{\text {di }}$ while too large a gap results in the occurrence of tilting. The Beta-Il experiment gave similar resulrs. ${ }^{11}$

\section{PERFORMANCE COMPARISON}

In the above comparison between 10 and 20 connectors, it was found that identical external parameters (e.g., bank voltage, EF current, etc.) led to different values of the major radius at the $\theta=5^{\circ}$ times. Therefore, direct comparison of these growth rates includes major radius scaling effects, which are complicated by unknown tux core stabilization effects. Here the stabilization techniques are compared as a function of major radius.

The data shown in Figs. 8-10 were obtained by fixing all external parameters (in particular, $25.5 \mathrm{kV}$ for the TF bank voltage) and changing the external equilibrium field strength. The results ase then plotted as a function of the magnetic axis major radius obtaised at the $\theta-5^{\circ}$ time.

Figure 8 shows the measured major radius dependence of the detachment value, $\left(\psi_{0}-\psi_{\text {core }}\right) / \psi_{0}$, which describes the fraction of poloidal flux that is detached from the flux core. A completely formed spheromak has a detachment value equal to (or greater than) one. This occurs for major radii !ess than $\simeq 13.5 \mathrm{~cm}$. For this reason, it is desired that stabilizing structures work well for major radii in the range $13-14 \mathrm{~cm}$. Notice that for major radii of $16-19 \mathrm{~cm}$, typical of many experimental runs, the detachment value ranges from 0.4 to 0.1 . In this range, lux core effects on tilting are experted to be significant.

Figure 9 shows the measured major radius dependence of the growth rate for the passive systems discussed in this paper. In addition, available "figure 8 " and 
"saddle" coil data have been included. In all cases, the growth rate increases with decreasing major radius, usually very rapidly below a certain radius. The improvement obtained with the various techniques is clear from the figure. In particular, the addition of the 20 connectors has the biggest effect for radit less than $\simeq 17 \mathrm{~cm}$, at which point the the growth raie for plain wagon wheels begins increasing rapidly. The 20 connector case has allowed major radius operation at $13 \mathrm{csn}$ while maintaining a moderately small growth rate.

The other parameter of interest is the tilting onset time, or, the useful quiescent spheromak lifetime. Figure 10 shows the "lifetime", defined as the time from breakdown of the neutral gas until $\theta$ reaches $10^{\circ}$, which includes the $\sim 30$ to $40 \mu$ s formation time. Again, the figure clearly shows the improvement obtained with the various techniques. With the 20 connectors, the spheromak "lifetime" is maintained at $\simeq 100 \mu$ s for discharges with a $13-\mathrm{cm}$ major radius, a significant improvernent over other techniques.

\section{iV. PERTURBED CURRENT PATTERN}

A direct measurement of the perturbed current patterns in the passive structures would reveal important information on the underlying mechanism of stabilization. In particular, line-tying effects should produce a perturbed current pattern which is different from a purely inductive pattern. ${ }^{24}$ Studying the detajls of the current pattern should confirm the presence, or absence, of line-tying stabilization in the various types of stabilizing structures. The wagon wheels, with and without connectors, are good candidates for this measurement because Rogowski coils can be added to the spokes. In this section, the results from such a measurement are discussed.

The positions of the Rogowski coils on the stabilizing structure are shown in Fig. 11. In the case of plain wagon wheels, 14 coils were used. Four additional 
coils were added to monitor the current flowing in the connectors, when the connectors ver installed. These 4 coils were mounted in different configurations during two difierent experimental runs, as shown in the figure.

Line-tying currents should make $\vec{\nabla} \cdot \vec{J}_{\text {stabilizing coil }} \neq 0$, and the goal is to lucate positions on the stabilizing coil where this occurs. For this reason, the Rogowski coil configurations were chosen to give detailed structure within a toroidal wedge. The size of the wedge was limited to 108 degrees, keeping the number of Rogowskj coils down to a manageable level.

The grid defined by the Rogowski coils divides the stabilizing rods into nodes where Kirchhoff's current law can be applied. A node can consist of two joined rods plus an assumed plasma source, or simply the two ends of a single rod plus an assumed plasma source. In either case. the sum of all current into a node must be zero, so the plasma source current for that node can be determined and subsequently jdentified as line-tying current.

During the experiments without the connectors, the reproducibility of the tilting toroidal phase was unusually poor. This randomness of phase was particularly fortuitous for the purpose of this experiment. The actual toroidal phase on a given discharge can be determined from the internal probe data (in combination with extensive experience with the tilting mode in this machine). By firing many discharges with random phases, the data from the Rogow:ki coils can be used to reconstruct the current pattern over the entire wheel, instead of the $108^{\circ}$ wedge. A total of 34 discharges from an experimental run using plain wagon wheels were binned into 8 bins, each of which covers a phase of $\pi / 4$. Data within a bin were averaged.

The reconstructed rvrrent pattern is shown in Fig. 12. The restults indicate that line-tying current can be divided into two classes: equilibrium current and perturbed current. The equilibrium line-tying current arises from the steady- 
state interaction of the stabilizers and the spheromak. Experimentally, this c-1rrent enters the wheel near the center, flows radially outward, and exits the wheel at the edge. This is expected since some poloidal flux bulges outside the wheels (i.e., above and below), and poloidal current on these surfaces flow predominately in the highly conductive copper instead of the comparatively resistive plasma above (below) the top (bottom) wheel. The equilibrium current flowing in the wheel is found to be $\simeq 21 \%$ of the total spheromak poloidal current a! $\mathrm{t}=54 \mu \mathrm{s}$, and drops to $\simeq 10 \%$ at $\mathrm{t}=76 \mu \mathrm{s}$.

In this study, the line-tying current pattern that develops during the tilting phase of the spheromak is more interesting. The resulting perturbed current pattern is determined by subtracting the tojoidally averagnd data (at each radius position on the wheel) from the original data. The results are shown in Fig. 12 for $t=80$ and $90 \mu \mathrm{s}$. The diagnosed wheel is at $+11.5 \mathrm{~cm}$, the axis of tilting is "out of the page" for the contour plots of constant $B_{t}$ (toroidal field), toroidal plasma current to the right of the symmetry axis (in the contour plots) is directed "out of the page," and the axis of tilting is in the "6:00" direction for the wheel plots.

Perturbed current enters the radial spokes on one toroidal side, flows across the wheel through the inner hoop, and exits the wheel on the other toroidal side (see Fig. 12). Since the current enters the coil from the plasma, and exits to the plasma, it is identified as line-tying current, as defined in this article.

The observed pattern is in the direction required to produce a horizontal magnetic field which retards the tilting motion. During the tilting phase, the current in the outer hoop remains primarily axisymmetric (subtsaction of the toroidally averaged outer hoop current leaves nearly zero perturbed current, as seen in Fig. 12). There is little evidence of a "figure 8 " shaped inductive current pattern. 
When the connectors are installed, the equilibrium tine-tying current enters the wheel near the center, as without the connectors. However, the current does not leave the wheel at the edge but continues in the connectors to the other wheel (exiting near the center; odd symmetry about the midplane). This current is $\approx 49 \%$ of the total spheromak poloidal current at $\mathrm{t}=64 \mu \mathrm{s}$, and drops to $\simeq 32 \%$ at $t=84 \mu \mathrm{s}\left(\theta=10^{\circ}\right)$.

The reproducibility of tilting toroidal phase angle was exceptionally good during these experimental runs, preventing the use of the binning procedure described above. Initially, the four Rogowski coils on the connectors were positioned to test current conservation at the nodes where the connectors meet the outer hoop (position 1 in Fig. 11). Once it was confirmed that line-tying current does not appear at these nodes, the four coils were moved $\simeq 180^{\circ}$ (position 2 in Fig. 11). The results show that perturbed current flows across the the wheel as in the previous case, but returns to the other wheel along the connectors, instead of through the plasma. The data indicate that the perturbed cursent flows entirely in the rods, and thus, should not be identified as line-tying current. The measured current is an ideal MHD inductive type of cursent. Sote that the inductive current pattern is, again, not "figure 8" shaped, but rather, one which agrees with the prediction shown in Fig. 5.

\section{LINE-TYING MODEL}

The results from the previous section demonstrate that the wagon wheel stabilizer invo' "es primarily a line-tying effect, while the addition of connectors results in ideal MHD inductive stabilization. In the latter case, this inductive stabilization should be described by the usual ideal MHD modeling, and the results are in reasonable agreement with predictions. However, the line-tying effect is usually not included in idea] MHD modeling. In this section, a model 
of tilting including line-tying is presented. A comparison with results from previous calculations is made.

A qualitative picture of line-tying stabilization is given in Fig. 13. The spheromak tilting motion tends to induce current in the wall as shown in Fig. 13(a). Notice that the induced current is unipolar across ejther wall. Now examine the situation rotated toroidally by 90 degrees, as shown in Fig. 13(b). Without line-tying, the induced wall current would not have an optimal return path. Charge separation would occur, and the current would stop fowing. The induced emf would then appear across the sheath region at the plasma-wall boundary, and current would then be collected from the plasma vic the same mechanism by which Langmuir probes collect current. This, then, provides a return path for the riginal incuced current. The resulting cusrent pattern is similar to the pattern prewicted for a fully enclosing spherical shell, shown in Fig. 5. Since the fully enclosing shell permits all possible cursent patterns, the one that actually occurs must be the optimal pattern for stabilizing the tilt.

A comment should be made about magnetic field lines that, presumably, are frozen into the conducting wall (see Fig. 3). Assuming tiut the plasma in and around the sheath region is cold and dirty, the reconrection time scale will be short enough to allow field lines in the plasma to reconnect constantly (forced reconnection) with neighboring field lines frozen in the wall. In this way, field lines can mo"e along the wall, as necessary, without actually dragging the frozen field line segments through the wall (as was previously implied in Fig. 3). In the case of the wagon wheel, this mechanism is assumed to allow field lines to "pass through" the conducting rods (if necessary).

In this model, the magnitude of perturbed current collected through the sheath is determined by the sheath resistance. A simple estimate of the driving voltage and sheath resistance is now used to predict the magnitude of the 
perturbed current. This estimate is then incorporated into the ring model of tilting to predict the growth rate in the presence of line-tying effects,

The relevant voltages are shown in Fig. 13(b). The wall is assumed to be perfectly conducting. The plasma has a perturbed electric field $\left(\vec{E}_{p}\right)$ approximated by

$$
\vec{E}_{p}+\frac{1}{c} \vec{V} \times \vec{B}=0,
$$

where $\vec{V}$ is the tilting velocity. This electric field, shown in Fig. 13(b), produces a voltage drop in the $F$ asma given by $\Phi=\Phi_{2}-\Phi_{1}$. The sheath drop at each end is given by $\Phi_{2}-\Phi_{\text {wall }}$ and $\Phi_{1}-\Phi_{\text {wall, }}$ and the situation is analogous to the double Langmuir probe. ${ }^{42}$ The current collection is estimated by the usual double Langmuir probe voltage characteristic ${ }^{43}$

$$
I_{\text {l.t. }}=A J_{i o n} \tanh \left(\frac{e \Phi}{2 k T_{e}}\right),
$$

where $I_{l . t .}$ is the line-tying current, $A$ is the effective collection area of the wall, $J_{i o n}$ is the ion saturation current density given by

$$
J_{\text {ion }} \simeq 0.6 n_{i} q_{i}\left(\frac{k T_{e}}{m_{i}}\right)^{1 / 2},
$$

and $\Phi=\Phi_{2}-\Phi_{1}$ is given by

$$
\Phi=-\int \vec{E}_{p} \cdot d \vec{\tau}
$$

The perturbed current flowing in the wall will produce a perturbed magnetic

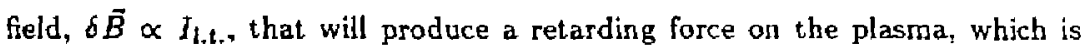
included in the equation of motion

$$
\rho \frac{d \bar{V}}{d t}=\vec{J} \times\left(\bar{B}_{0}+\delta \bar{B}\right),
$$

whese $\vec{J}$ is the plasma current density, $\vec{B}_{0}$ is the vacuum equilibrium "vertical" field (the driving force for the tilt mode), $\delta \vec{B}$ is the perturbed magnetic field due to the line-tying current, and $\rho$ is the plasma mass density. 
An estimate of the growth rate is made by finding the torque on the plasma, gives by

$$
\vec{\tau}_{t i l t}=\frac{1}{c} \int_{\substack{\text { plasma } \\ \text { volume }}} \vec{r} \times(\vec{J} \times \vec{B}) d^{3} x .
$$

In the ring model, this is approximated by a large aspect ratio toroidal current ring, which gives

$$
\left.\tau_{t i l t}=\frac{\pi}{c} R_{J}^{2} J_{D} \mid\left(B_{0}-\delta B_{z}\right) \sin \theta-\delta B_{z} \cos \theta\right],
$$

where $R_{J}$ is the major radius of the current ring, $I_{0}$ is the toroidal current, and $\theta$ is the tilt angle. In this paper, the vacuum "vertical" field is assumed straight $\left(n_{\mathrm{i}}=0\right)$ and in the $\mathrm{Z}$ direction. The perturbed magnetic field has both vertical and horizontal components; however the vertical component is zero at $\theta=0$ due to up/down symmetry. Thus, the $\delta B_{2}$ term is nonlineas in theta.

The torque is balanced by the moment of inertia $\left(I_{x x}\right)$

$$
I_{x x} \bar{\theta}=\tau_{t i l t}
$$

with

$$
I_{x x}=\frac{1}{2} M R_{J}^{2}\left[1+\frac{5}{4}\left(\frac{a}{R_{J}}\right)^{2}\right],
$$

where $M$ is the plasma mass, approximated by $\rho 2 \pi^{2} a^{2} R_{j}$, and $a$ is the minor radius of the current ring.

The driving electric field is roughly

$$
\left|E_{p}\right|=\frac{1}{c} R_{J} \dot{\theta}\left\langle B_{z} \sin \theta-B_{x} \cos \theta\right),
$$

where $B_{x}$ and $B_{z}$ are the magnetic field compoilents at the wall. The driving voltage is then estimated by

$$
\Phi=\frac{1}{c} R_{J} L \dot{\theta}\left\langle B_{z} \sin \theta+B_{x} \cos \theta\right\rangle,
$$

where $L$ is the effective integration length between points 1 and 2 in Fig. 13(b), and $\langle\ldots\rangle$ indicates the average value along the wall. 
By combining Eqs. (3) - (6), and defining

$$
\bar{\delta}_{x} \equiv \delta B_{x} / I_{l . t .} \quad, \quad \bar{\delta} \bar{B}_{z} \equiv \delta B_{z} / I_{l . t .}
$$

the following equation for theta is obtained

$$
\vec{\theta}=\gamma_{0}^{2} \sin \theta\left\{1-\frac{\cos \theta}{\sin \theta} \alpha \tanh (\xi \dot{\theta})\right\} .
$$

The following definitions have been introduced:

$$
\begin{gathered}
\xi=\left(R_{J} L B_{\text {edge }}\right)\left(2 \varepsilon \frac{k T_{e}}{e}\right)^{-1} \\
\alpha=\frac{\overline{\delta B}}{B_{0}} 0.6 A n_{e} e\left(\frac{k T_{e}}{m_{i}}\right)^{1 / 2} \\
\overline{\delta B}=\overline{\delta B}_{z}+\overline{\delta B}_{z} \frac{\sin \theta}{\cos \theta}
\end{gathered}
$$

and

$$
\gamma_{0}^{2}=\frac{\pi}{c I_{x x}} I_{0} R_{J}^{2} B_{0}
$$

where $B_{e d g e}=\left\langle B_{z} \sin \theta+B_{I} \cos \theta\right\rangle$. Note that $\gamma_{0}$ is the customary unstabilized ring model growth rate. ${ }^{20}$

Equation $(7)$ is a complicated highly nonlinear equation, especially since $\xi, \alpha$, $\overline{\delta B}$, and $\gamma_{0}$ are dependent on time and/or theta. In this paper, two approaches are taken. A numerical time integration is described in Sec. V-A. Progress can be made, however, by writing

$$
\begin{gathered}
\gamma \equiv \frac{1}{\theta} \frac{\partial \theta}{\partial t} \\
\ddot{\theta}=\theta \gamma^{2}+\frac{1}{2} \theta^{2} \frac{\partial \gamma^{2}}{\partial \theta}
\end{gathered}
$$

In terms of this definition, the growth rate, including the line-sying effect, is given by

$$
\gamma^{2}=\gamma_{0}^{2} \frac{\sin \theta}{\theta}\left\{1-\frac{\cos \theta}{\sin \theta} \alpha \tanh (\xi \gamma \theta)\right\}-\frac{1}{2} \theta \frac{\partial \gamma^{2}}{\partial \theta}
$$


Notice that the $\partial \gamma^{2} / \partial \theta$ term tends to moderate changes in $\gamma$ versus $\theta$ because of the minus sign. For the remainder of this paper, discussion of Eq. (12) will ignore this term.

Equation (12) has several interesting limits. In the limit $\xi \gamma \theta \ll 1$, the hyperbolic tangent linearizes, giving

$$
\quad \gamma^{2} \simeq \gamma_{0}^{2}(1-\alpha \xi \gamma) \text {, }
$$

taking $\sin \theta \simeq \theta$, and $\cos \theta \simeq 1$. If $\alpha \rightarrow 0$, corresponding to the absence of line-tying, Eq. (13) gives $\gamma=\gamma_{0}$. On the other hand, if line-tying is extremely strong, corresponding to $\alpha \rightarrow \infty$, Eq. (13) gives

$$
\gamma \simeq(\alpha \xi)^{-1}
$$

and the requirement $\xi \gamma \theta \ll 1$ is satisfied for $\theta \ll \alpha$.

In the othes limit of $\xi \gamma^{\theta} \gg 1$, the hyperbolic tangent saturates, and Eq. (12) gives

$$
\gamma=\gamma_{0}\left(1-\alpha_{z}\right)^{1 / 2} \text {. }
$$

taking $\sin \theta \simeq \theta \simeq 1$, and $\alpha_{z}$ is the component of $\alpha$ die to the $\delta B_{z}$ lerm.

These limits demonstrate two important properties of this model. According to Eq. (14), line-tying stabilization can never completely stabilize the tilting mode. Physically, $\alpha$ is limited to a finite value, typically of order 1 . But even formally, $\alpha \rightarrow \infty$ is not suffictent to make $\gamma^{2} \leq 0$. On the other hand, a modest level of line-tying is sufficient to make a noticeable reduction in the growth rate. even in the worst case of Eq. (15). For example, if $\alpha_{z}=0.7$ and $\theta \geq(\xi \gamma)^{-1}$. there is a $45 \%$ reduction in growth rate.

The effect of the explicit $\theta$ dependence in Eq. (12) is demonstrated in Fig. 14(a). The quantity $\gamma_{\theta}$ is the solution of Eq. (12) with fixed values of $\alpha=0.313, \xi=32.9$, and $\%_{0}=0.766$. Note that this curve contains both limits discussed above; Eq. (14) determines the value of $\gamma_{\theta}$ for $\theta \leqslant 10^{\circ}, \mathrm{Eq}$. (15) 
determines the value of $\gamma_{\theta}$ for $\theta \gtrsim 50^{\circ}$, and the transition region corresponds to the onset of saturation of $\tanh (\xi \gamma \theta)$. (The quantity $\gamma_{a}$ plotted in Fig. 14(a) will be described in Sec. V-A.)

Previous numerical MHD calculatjons, both $2-D^{22,23}$ and $3-D,{ }^{24}$ have been carried out, including line-tying effects. As mentioned in the introduction, these calculations are in qualitative agreement with experiment in the sense that a large line-tying effect has been identified in both cases. In particular, they agree with the experiments in Proto $S-1$, and $S-1,33,44$ with regard to the conditions necessary for strong effects (for example, Sec. III-A). The disagreement with experiment arises from the eariier prediction that line-tying can completely stabilize the tilting mode. In such calculations, idealized plasma-conducting wall boundary conditions are employed and the sheath is neglected. This corresponds to setting $\alpha \equiv \infty$ in Eq. (14). In that approximation, the present model and previous calculations agree; as long as $\xi$ in Eq. (14) is non-zero, there is complete stabilization. The important contribution of the model in this paper is the identification of at least one mechanism through which the effectiveness of line-tying will be reduced.

\section{A. COMPARISON WITH EXPERIMENT: TIME BE- HAVIOR}

The model presented in Sec. $\mathrm{V}$ is admittedly a simplification of spheromak tilting dynamics, but it is of interest to see how well Eq. (7) agrees with the experimenta! measurements.

Based on the measured current pattern shown in $\bar{r}$ ig. 12, the function $\delta \vec{B}(\overrightarrow{\mathcal{I}})$ can be estimated using the Biot-Savart law, where the integration includes only the current elements in the wall. The value of $\overrightarrow{\delta B}$ is then determined by dividing $\delta \vec{B}(\vec{x})$ by the maximum value of perturbed current crossing from one half of the wheel to the other (which occurs at the inner hoop). The results are given as a 
function of 8 , for a fixed major radius of $11.7 \mathrm{~cm}$, in Table 2 .

As expected, the value of $\overline{\delta B}$ varies with $\theta$, thus, $\alpha$ also varies with $\theta$. The effect on Eq. (12) of introducing this $\theta$ dependence in Eq. (9j, keeping all othei parameters fixed, is shown in Fig. 14(a) bv the quantity $\gamma_{\alpha}$. The values of $\gamma_{0}$, $\xi$, and $\alpha$ used in Fig. 14(a) are based on the experimental parameters at the $\theta=5^{\circ}$ time point, which are as follows:

$$
\begin{aligned}
& I_{0}=38 \pm 2 \mathrm{kA} \quad, \quad B_{0}=360 \pm 10 \mathrm{G} \\
& R_{J}=11.7 \pm 0.3 \mathrm{~cm} \quad: \quad B_{\text {edge }}=360 \pm 40 \mathrm{G} \\
& T_{\mathrm{e}}=7.5 \pm 1.5 \mathrm{eV} \quad, \quad n_{\mathrm{e}}=(1.2 \pm 0.1) \times 10^{.4} \mathrm{~cm}^{-3} \text {, } \\
& \gamma_{0}=0.77 \pm 0.05 \mu \mathrm{s}^{-1}, \gamma_{\text {measured }}=0.098 \pm 0.010 \mu \mathrm{s}^{-1},
\end{aligned}
$$

and by taking

$$
A \sim 340 \mathrm{~cm}^{2}, L \sim 11.7 \mathrm{~cm}
$$

which gives

$$
\xi \sim 33 \mu \mathrm{s}, \alpha\left(5^{\circ}\right) \sim 0.3 .
$$

The values of $I_{0}, R_{S}, B_{e d g e}$, and $\gamma_{\text {measured }}$ are based on the internal magnetic measurements. The values for $T_{e}$ and $n_{e}$ (obtained from Thomson scattering) are based on an average of $\approx 35$ shots, where the laser was fired between $t=60$ and $90 \mu \mathrm{s}$. The extreme difference in behavior of $\gamma_{\theta}$ and $\gamma_{\alpha}$ is due to the fact that $\alpha$ increases in magnitude by more than a factor of 8 going from $1^{\circ}$ to $60^{\circ}$.

A comment should be made about the quoted value of $F_{J}$, which is the major radius corresponding to the peak toroidal current density (as indicated by the peak toroidal field, assuming a Taylor equilibrium ${ }^{45}$ ). For a spneromak, the value of $R_{J}$ differs from the value of the magnetic axis major radius $\left(R_{m . a}\right)$, because of the small aspect ratio. This discharge has a measured value of $R_{\text {m.a. }}=15.0 \pm 0.5 \mathrm{~cm}$. See the appendix for a further discussion of $R_{m . a}$. versus $R_{J}$.

The plot of $\gamma_{a}$ in Fig. 14(a) assumes that the plasma parameters are constani thuoughout the tilting phase. Experimentally, this is not true, as shown in 
Table 2. The additional effects on Eq. (12) due to the changing plasma pasameters are shown in Fig. 14(b), where the parameters $\gamma_{0}, \alpha$, and $\xi$ are evaluated according to Table 2, assuming $R,, L, A, T_{e}$, and $n_{e}$ are fixed in time. Comparison of the curves in Figs. 14 (a) and (b), for $A=340, L=11.7$, show that the additional effects are not large. (For the remainder of this subsection, $A$ will be in units of $\mathrm{cm}^{2}$ and $L$ in units of $\mathrm{cm}$.) Most of the additional effect is due to changes in $B_{e d g e}$, which enters in $\xi$. The incremental effect of adding the time-dependent $\gamma_{0}$ produces a maximum change of $5 \%$ (average change of $1.5 \%)$ in the curve. This implies that the value of $\gamma$, for tisise parameters, is basically determined by Eq. (14), which is independent of $\gamma_{0}$. For comparison, the result using $A=200, L=\mathbf{2 3 . 4}$ is also presented in the figure (see below).

An alternate approach to solving Eq. (7) is numerical integration using a finite difference scheme, where

$$
\begin{gathered}
\dot{\theta} \approx \frac{\theta_{n+1}-\theta_{n-1}}{2 \Delta t}, \\
\ddot{\theta} \approx \frac{\theta_{n+1}-2 \theta_{n}+\theta_{n-1}}{(\Delta t)^{2}},
\end{gathered}
$$

and employing the known time dependence in the plasma parameters given in Table 2. The integration starts at $t=56 \mu \mathrm{s}$, with $\theta_{\text {initial }}=1^{\circ}$ and $\gamma_{\text {initial }}=\gamma_{\text {measured }}$. The integration stops when the condition $\theta \geq 60^{\circ}$ occurs. Both the predicted $\theta(t)$ and the magnitude of the line-tying current, $I_{l . t}(t)$, are calculated. Comparison with the experimentally measured $\theta(t)$ and $I_{\text {l.t. }}(t)$ is made in Fig. 15.

The combination $A=340, L=11.7$ is taken as a "best fit" of the model to the measured growth rate. Notice the clear indication of hyperbolic tangent saturation in Fig. 15(b) ( $A=200, L=23.4)$ after $88 \mu \mathrm{s}$, pinning the current at $6.3 \mathrm{kA}$.

An upper limit on $A$ is determined by the actual surface area of the cop- 
per rods, which gives $A \leq 430$. However, it is well known that the effective collection area of Langmuir probes (in a magnetic field) can be very different than the geometric surface area. ${ }^{40}$ The effective collection area is estimated at $A \simeq 200 \pm 20 \mathrm{~cm}^{2}$, based on the magnitude of the measured current.

The curves of Fig. 15 indicate that the combination $A=340, L=11.7$ fits the experimental data much better (notice in particular the predicted decrease in current after $93 \mu \mathrm{s}$ ) than $A=200, L=23.4$, with the following proviso. The current plotted in Fig. 15(b) for $A=340$ has been reduced by the factor 200/340 from the actual predicted current. This is equivalent to maintaining the area at $200 \mathrm{~cm}^{2}$, and increasing $\overline{\delta B}$ by the factor $340 / 200$ (see Eq. (9)). Since this discharge has a. detachmeni value of $\approx 0.75 \pm 0.05$ (at the $\theta=5^{\circ}$ time point), the anomaly can be attributed to a flux core stabilization effect, which has the effect of increasing the magnitude of the perturbed stabilizing magnetic field. An ad hoc way of including the flux core is to inc rease the assumed magnitude of $\overline{\delta B}$ (a complete calculation of $\alpha$ fux core and $\xi$ fiux core is beyond the scope of this рарег). As shown in Fig. 15, a 70\% increase in $\overline{\delta B}$ to account for the flux core results in extremely good agreement between the line-tying model and the experimental measurements of $\theta(t)$ and $I_{l . t}(t)$. This implies that the flux core is providing $41 \%$ of the total stabilization force for this discharge.

It is interesting to compare the sheath resistance to the wall resistance, and to the bulk plasma resistance. The sheath resistance, assuming a linearized hyperbolic tangent, can be written as

$$
R_{\text {sheath }}=2.13 \times 10^{13} T_{\mathrm{e}}^{1 / 2} n_{\mathrm{e}}^{-1} A^{-1} \mathrm{Ohm} .
$$

For the values of $T_{e}$ and $n_{e}$ given in Eq. (16), and $A=340 \rightarrow 200$, the sheath resistance is $R_{\text {sheath }}=1.4 \rightarrow 2.4 \mathrm{~m} \Omega$. The resistance of the $0.51-\mathrm{mm}$ stainless steel wall is roughly $1.0 \mathrm{~m} \Omega$. Thus, the sheath is roughly twice as resistive as the thin stainless steel wall. The resistance of the path from one wall to the 
other through the plasma is roughly $0.25 \mathrm{~m} \Omega$ (see Fig. 13), estimated by taking a path $12 \mathrm{~cm}$ by $56 \mathrm{~cm}$ in cross-sectional area (major radius; $12 \mathrm{~cm}$ to $24 \mathrm{~cm}$. toroidal direction: $18 \pi \mathrm{cm}$ ), ajd $23 \mathrm{~cm}$ in length (distance between the walls), and using $Z_{\text {eff }}=1.5$. To summarize, the sheath is the dominant resistance, even with the resistive stainless steel wall. This explains the lack of a significant difference in growth rate between the discharges with thin stainless steel wall and wagon wheel stabilizers.

\section{B. COMPARISON WITH EXPERIMENT: CURRENT SCALING}

The previous subsection demonstrates the good agreement between the linetying model and the experimental measurements for one isoiated case. Further support for this model is obtained by comparing the predicted dependence of the growth rate on the plasma current with experiment.

The previous subsection showed that, for the parameters of this experiment, Eq. (14) is a reasonably accurate approximation to the solution of Eq. (7). Therefore, Eq. (14) can be used to determine the predicted scaling with current. Combining Eqs. (8) and (9), the growth rate can be written as

$$
\gamma_{l . t .}=2.13 \times 10^{15}\left\{\frac{B_{0}}{B_{\text {edge }}}\left(\overline{\delta B} A L R_{J}\right)^{-1}\right\} T_{e}^{1 / 2} n_{e}^{-1} \mu \mathrm{s}^{-1} .
$$

Notice that $B_{0} \propto J_{0}$ and $B_{e d g e} \propto I_{O}$, and the pirameters $\bar{\delta} \bar{B}, A, L$, and $R_{J}$ are geometrical factors. Thus, Eq. (17) is explicitly independent of the plasma current. The only way for a current dependence to enter is through the variation in $T_{e}$ and $n_{e}$ with plasma current. This is very different from the usual ideal MHD scaling, which can be written as

$$
\gamma \text { MHD } \propto I_{0} R_{j}^{-1} n_{e}^{-1 / 2} .
$$

The experimental current dependences of $T_{e}$ and $n_{\mathfrak{e}}$, as determined by Thomson scattering, are shown in Fig. 16. Each data point represents an average of 
20-35 shots, where the laser is fired between $t=60$ and $90 \mu \mathrm{s}$. The magnetic axis major radius for these discharges was $12.5 \pm 0.5 \mathrm{~cm}$, and the fill pressure was $10 \mathrm{mTorr}$ of hydrogen. Over the range $50 \leqq I_{0} \leqslant 95 \mathrm{kA}$, the plasma temperature is nearly constant and the density is roughly linear with plasma current. Notice that this implies the growth rate given by the line-tying model should decrease with increasing plasma current. This is, in fact, observed.

Since the plasma temperature is reasonably independent of plasma current, Eq. (17) predicts that $n_{e} \gamma$ should also be independent of current (for fixed $R_{J}$ ). Ideal MHD predicts $n_{e} \gamma \propto I_{0} n_{e}^{1 / 2}$. Figure 17(a) shows the experimental growth rate scaling for discharges with $0.51-m m-t h i c k$ siainless steel wall stabilizers. A case with 10 spoked wagon wheel stabilizers is also included. The discharges in this figure have magnetic axis major radii of $17.5 \pm 0.5 \mathrm{~cm}$. The experimental value of $n_{e} \gamma$ is reasonably independent of plasma current over the available range of $35 \leqslant I_{0} \leqq 100 \mathrm{kA}$, as predicted by $\mathrm{Eq}$. (17). This range is large enough to demonstrate a significant discrepancy with respect to a scaling of $I_{0} n_{\varepsilon}^{1 / 2}$, as shown in the figure.

At this point, the comparison between wagon wheels with 10 and 20 spoises can be made. Figure 17(b) shows the values of $n_{e} \gamma$ for two discharges with $15.5 \pm 0.5 \mathrm{~cm}$ major radii. The value of $n_{\mathrm{e}} \gamma$ for the 10 spoked case is 1.9 times greater than $n_{e} \gamma$ for the 20 spoked case. Equation (17) predicts $n_{e} \gamma \times A^{-1}$, and it is reasonable to expect that the effective collection area of a wheel is proportional to the number of spokes. Therefore, the difference in measured growth rates for the two cases is in agreement with the line-tying model.

The experimental scaling obtained with the full enclosure (20 connectors) is shown in Fig. 18 for two values of magnetic axis major radius $-16 \mathrm{~cm}$ and $12 \mathrm{~cm}$. As stated previously in Sec. III-D, line-tying to the flux core is expected to be important in the 16-cm-radius discharges. The data in Fig. 18 (for 
$16 \mathrm{~cm}$ ) are consistent with either the line-tying model or jdea! MHD scaling and is probably a combination of both. However, the $12-\mathrm{cm}$-radius discharges are completely detached from the flux core, which should no longer play an important stabilization role. In addition, the results in Sec. IV indicate that the full enclosure provides ideal MHD inductive stabilization. The data in Fig. 18 show that the experimental values of $n_{\mathrm{e}} \gamma$, for $12-\mathrm{cm}$-radius discharges, vary with plasma current, and the scaling is in reasonable agreement with ideal MHD. This provides further support to the conclusion that the full enclosure doe rot rely on line-tying.

\section{CONCLUSIONS}

Developing effective schemes for stabilizing the tilt mode, while maintaining compatibility with the S-1 inductive formation scheme, has been (and may continue to be) a difficult problem. It has been shown that the performance of pas. sive stabilization can be significantly improved by allowing designs which have some coupling with the flux core. The coupling allowed in these experiments has a minimal impact on formation efficiency. In fact, the best discharges have higher toroidal current for a given bank voltage than discharges with ( $n=0$ ) noncoupling stabilizers ("figure 8 " and "saddle" coils). This occurs because previous discharges became unstable before the driving flux swing was complete. The improved stability in the best cases can maintain stable spheromaks throughout the entire driving flux swing, resulting in higher current. In addition, the improved stability allows operation with smaller major radii, which increases the toroidal current through compressive effects. Peak toroidal currents of $105 \mathrm{kA}$ at $25.5 \mathrm{kV}$ (on the TF bank) have been obtained with both wagon wheels and the full enclosure, compared to $60-70 \mathrm{kA}$ at $26 \mathrm{kV}$ obtained with previous $(\mathrm{n}=0$ ) noncoupling stabilizers. 
Reasonably large axisymmetric equilibrium line-tying currents were observed in both the "wagon wheel" and "full enclosure" stabilizers. These currents were largest in the "full enclosure" ( $\$ 49 \%$ of the spheromak poloidal current). The level of this equilibrium current is presumably determined, in part, by the ratio of stabilizer electrical conductivity to plasma conductivity. In this experiment, that ratio is very high. The level of equilibrium current inay also be enhanced berause the wheel lies in a plane, thereby producing a structure more suitable for an oblate plasma shape, while the actual plasma is prolate. Thus, unnecessary amounts of flux bulge out the top and bottom. It is possible that a more sophisticated (nonplanar) shape for the wagon wheel would reduce the equilibrium line-tying current without sacrificing effectiveness. These considerations will certainly affect the future usefulness of the "wagon wheel" and "full enclosure" coil structures.

Aside from verification of S-1 formation, the main purpose of the Proto $\mathbf{S - 1}$ devices has been to provide a test bed for proposed tilt stabilization schemes, which may be used in larger spheromak devices. In this light, it is important to Inderstiu, d the underlying physical mechanisms providing stabilization, so that fut ure usefulness can be evaluated. In the course of this study, experimental evidence for a line-tying effect was found during the solid wall experiments. Further evidence was obtained by comparing the wagon wheel results with the results using the thin stainless steel wall. It appears that wall resistance is not the limiting factor.

Important characteristics of the line-tying effect were ob.ained by actual measurement of the perturbed current pattern in the vagois whees stabiiizer. The results indicate that the perturbed cursent is not contained within the stabilizer, but leaves the stabiiizer and goes into the plasma. This type of pattern was predicted by 3-D simulations. ${ }^{24}$ However, the theoretical calculations 
predicted complete stabilization, which is not observed experimentally. Having confirmed the character of the perturbed current pattern, it is clear that the resistance of the plasma sheath at the wall must be accounted for. For this parpose, the line-tying model in Sec. $\mathrm{V}$ was developed. The comparison with experimental results in Secs. V-A and V-B shows good agreement between predicted and observed growth rates. In addition, the model explains an otherwise puzzling result; higher current discharges tilt more slowly. This is due to increased plasma density at higher currents, which directly leads to increased effectiveness of the line-tying.

The proposed model can be summarized as follows. As the spheromak tilts, a voltage appears on the stabilizer, which causes current to flow. If a suitable return path is not available, charge will build up cn different areas of the wheel, and the voltage appears across the plasma sheath. At this poirt, current can be collected from the plasma via the same mechanism by which Langmuir probes collect current from a plasma, thus providing a return path for the initial induced current. In contrast, if the stabilizers are connected electrically to provide a return path, then the line-tying effect will be small. In essence, the connectors short out the line-tying effect.

From theoretical work, it is known that the fully enclosing solid wall is the nost effective passive stabilizer against tilting. Removal of essential sections would seriously limit the effectiveness of the wall; however, the line-tying effect can compensate for those sections, giving the necessary return path. This is an important feature of line-tying.

However, the disadvaisiages of line-tying stabilization (other than the obvious: increased impurities and enhanced parallel heat loss) become clear by examining the predictions of the model given here. First, the model predicts incomplete stabilization. Line-tying cannot even be used to supple!.ent other 
forms of stabilization to gain complete stability, because, in this model, tilting velocity is recessary to "turn on" the line-tying effects. In addition, the collected current is expected to be limited by the ion saturation current. The plasma on field lines that are near, or intersect, a metallic object will be cold and less dense than the central plasma, thus limiting the ion saturation current even in reactor-grade plasmas.

Equation (17) jmplies the growth rate is reduced by reducing $T_{e}$. Making $T_{e} \approx 0$ does not work, however, because Eq. (17) requires the condition $0 \ll a$, and $\alpha$ is proportional to $T_{e}^{1 / 2}$. Very cold plasmas will eventually violate the condition $\theta \ll \alpha$ (through experimental asymmetries or random motion), and Eq. (15) will describe the growth rate. High plasma temperature makes the condition $\theta \ll \alpha$ easy to satisify, but the growth rate will increase with $T_{e}$ as given by Eq. (17). Line-tying will thus be most effective for "intermediate" values of $T_{e}$.

Many tokamaks use active feedback stabilization to control axisymmetric vertical instabilities whose growth rates have been reduced by passive stabilization. ${ }^{47,4 \Delta}$ This suggests a similar combination of line-tying, and active feedback could be used to control the spheromak tilting mode. The tilting time scale should be increased to the millisecond regime to reduce the bandwidth and power requirements on an active feedback system. Line-tying may provide th. required reduction in large spheromak devices as indicated by examining the size scaling predicted by Eq. (17). Since $B_{0} / B_{\text {edge }}$ is independent of plasma size, and assuming $T_{e}$ and $n_{e}$ (edge values) are independent of size, and noting that $\overline{\delta B} \propto R^{-1}$ ( $R$ indicates the size of the spheromak), $A \propto R^{2}, L \propto R$, and $R_{J} \propto R$, the growth rate is proportional to $R^{-3}$ (the inverse volume of the spheromak). issuming the values $T_{e}=10 \mathrm{eV}, n_{e}=5 \times 10^{12} \mathrm{~cm}^{-3}, B_{0} / B_{\text {edge }}=1.5$ (which implies $B_{e d g e}$ is $25 \%$ of $B_{m, a}$, see the appendix), and scaling the values of $\overline{\delta B}$, 
$A, L$, and $R_{J}$ in Sec. V-A, Eq. (17) predicts a 1-ms tilting growth time for a 2.4-m magnetic axis major radius. This implies that large spheromaks may rely on line-tying for a substantial reduction in growth rate, and then use active Seedback to gain complete stability.

\section{ACKNOWLEDGMENTS}

I gratefully acknowledge useful suggestions by M. Yamada, S. Cowley, and R. Kulsrud.

I especially want to thank D.H. McNeill for the loan of the Thomson scattering equipment and for the expert advice he provided during the Thomson scattering measurements.

This work was supported by the C.S. Department of Energy Contract No. DE-ACO2-76-CHO-3073.

\section{APPENDIX : DISCUSSION OF $R_{\text {m.a. }}$ VERSUS $R_{3}$}

In Sec. $V$, the definition of $\gamma_{0}$ was developed assuming a large aspect ratio current ring. In that case, there is little difference between the major radius corsesponding to the peak toroidal current density and the major radius corresponding to the magnetic axis. However, the spheromak is a small aspect ratio configuration and there is a significant difference between the two radii. One way to gain consistency is to require the value of yo agree roughly with full 2-D ideal MHD free boundary calculations (i.e., wall at infinity), thus determining the effective radius to be used in calculating $\gamma_{0}$.

The growth rate for free boundary tilting, as given by ideal MHD stability codes such as ERATO ${ }^{49}$ and PEST, ${ }^{50}$ for an equilibrium suitable for comparison 
with this experiment, can be written as

$$
\gamma_{\text {MHD code }}^{2}=0.5 \frac{B_{m . a .}^{2}}{4 \pi \rho R_{m . a}^{2}},
$$

where $B_{m . d .}$ is the strength of the toroidal field at the magnetic axis, and $R_{m . a .}$ is the major radius of the magnetic axis.

The definition of $\gamma_{0}$ (Eq. (11)) can be transformed into the explicit scaling of Eq. (A1) by using the following relations" derived from the "classical spheromak $^{\text {n }}$

$$
B_{0}=0.38 B_{\text {m.a. }}, I_{0}=3.9 R_{\text {m.a. }} B_{\text {ma. }}, R_{J}=0.76 R_{\text {m.a. }} \text {, }
$$

where $B$ is in gauss, $R$ is in centimeters, and $I$ is in amperes. (Note: the discharge discussed in Sec. V-A, which has $B_{\text {m.a. }}=770 \pm 60 \mathrm{G}$, satisfies these selations to within $\approx 20 \%$.) Substitution of these relations into Eg. (11) gives the following form of $\gamma_{0}$

$$
\gamma_{0}^{2}=0.53\left(\frac{R_{m \cdot a}}{R_{J}}\right)^{3} \gamma_{M H D}^{2} \text { code }=1.2 \gamma_{M H D}^{2} \text { code } .
$$

Therefore, the use of $R_{J}$ is reasonably justued by the 2-D ideal MHD calculations. 


\section{REFEREINCES}

${ }^{1}$ D.C. Robinson, Plasma Phys. 13, 439 (1971).

${ }^{2}$ G. Miller, Phys. Fluids 28, 560 (1985).

${ }^{3}$ J.L. Srhwarzmeier, D.C. Barnes, D.W. Hewett, C.E. Seyler, A.I. Shestakov, and R.L. Spencer, Phys. Fluids 26, 1295 (1983).

${ }^{4}$ M.N. Rosenbluth and M.N. Bussac, Nucl. Fusion 19, 489 (1979).

${ }^{5}$ M. Okabayashi, P. Beiersdorfer, K. Bol, D. Buchenauer, M.S. Chance,

P. Couture, H. Eubank, H. Fishman, R. Fonck, G. Gammel, R. Goldston,

B. Grek, R.C. Grimm, W. Heidbrink, K. Ida, S. Ishida, K. Itami, R. Isso,

K. Jaehnig, S.C. Jardin, D. Johnson, R. Kaita, S. Kaye, H. Kugel, B. Leblanc,

J. Manickam, K. McGuire, D. Mueller, D. Monticello, W. Park, M. Reusch,

G. Rewoldt, G. Schmidt, S. Sesnic, W. Stodiek, H. Takahashi, W. Tang,

F. Tenney, R.B. White, A.M.M. Todd, M.W. Phillips, F.J. Helton,

J.M. Greene, and J.C. Wesley, in Proceedings of the Tenth International

Conference on Plasma Physics and Controlled Nuclear Fusion Research

(IA EA, London, 1984), p. 229.

${ }^{6}$ H.L. Berk and T.B. Kaiser, Phys. Fluids 28, 345 (1985).

${ }^{7}$ T.B. Kaiser and L.D. Pearlstein, Phys. Fluids 28, 1003 (1985).

'J. Kesner, Nucl. Fusion 25, 275 (198.5).

${ }^{9}$ D.A. D'lppolito and J.R. Myra, Phys. Fluids 27, 2256 (1984).

${ }^{10}$ Z.G. An. A. Bondeson, H. Bruhns, H.H. Chen, Y.P. Chong, J.M. Fiтn, G.C. Guldenbaum, H.R. Griem, G.W. Hart, R. Hess, J.H. Irby, Y.C. Lee, C.S Liu, W.M. Manheimer, G. Marklin, and E. Ott, in Proceedings of the Eighth International Conference on Plasma Physics and Controlled Nuclear Fusion (IAEA, Hrusse!s, 1980), p. 493.

${ }^{11}$ W.C. Turner, G.C. Goldenbaum, E.H.A. Granneman, J.H. Hammer, 
C.W. Hartman, D.S. Prono, and J. Taska, Phys. Fluids 26, 1965 (1983). ${ }^{12}$ T.R. Jarboe, I. Henins, H.W. Hoida, R.K. Linford, J. Marshall, D.A. Platts, and A.R. Sherwood; Phys, Rev. Lett. 45, 1264 (1980).

${ }^{13}$ A. Bondeson, G. Márklin, Z.G. An, H.H. Chen, Y.C. Lee, and C.S. Liu, Phys. Fluids 24, 1682 (1981).

${ }^{14}$ J.M. Finn, W.M. Manteimer, and E. Ott, Phys. Fluids 24, 1336 (1981).

15S.C. Jardin, M.S. Chance, R.L. Dewar, R.C. Grimm, and D.A. Monticello, Nucl. Fusion 21, 1203 (1981).

${ }^{16}$ P. Gautier, R. Gruber, and F. Troyon, Nucl. Fusion 21, 1399 (1981).

${ }^{17}$ J.H. Hammer, Nucl. Fusion 21, 488 (1981).

${ }^{18} \mathrm{~T}$. Sato and T. Hayashi, Phys. Rev. Lett. 50, 38 (1983).

${ }^{19}$ H. Bruhns, C. Chin-Fatt, Y.P. Chong, A.W. DeSilva, G.C. Goldenbaum, H.R. Griem, G.W. Hart, R.A. Hess, J.H. Irby, and R.S. Shaw, Phys. Fluids 26, 1616 (1983).

${ }^{20}$ C. Munson, A. Janos, F. Wysocki, and M. Yamada, Phys. Fluids 28, 1525 (1985).

${ }^{21}$ S.C. Jardin and U. Christensen, Nucl. Fusion 21, 1665 (1981).

22 J.M. Finn and A. Reiman, Phys. Fluids 25, 116 (1982).

${ }^{23}$ J.M. Finn, Phys. Fluids 27, 2973 (1984).

${ }^{24}$ T. Hayashi and T. Sato, Phys. Fluids 27, 778 (1984).

${ }^{25}$ A. Mohri, Jpn. J. Appl. Phys. 19, L686 (1980).

${ }^{26}$ R.N. Sudan and P.K. Kaw, Phys. Rev. Lett. 47, 575 (198I).

${ }^{27}$ C. Litwin, R.N. Sudan, and A.D. Turnbull, Phys. Fluids 27, 2791 (1984).

${ }^{2 B}$ G.C. Goldenbaum, J.H. Irby, Y.P. Chong, and G.W. Hart, Phys. Rev. Lett. 44, 393 (1980). 
${ }^{29}$ M. Yamada, H.P. Furth, W. Hsu, A. Janos, S.C. Jardin, M. Okabayashi, J. Sinnis, T.H. Stix, and K. Yamazaki, Phys. Rev. Lett. 46, 188 (1981). ${ }^{30}$ T.R. Jarboe, I. Henins, A.R. Sherwood, C.W. Barnes, and H.W. Hoida, Phys. Rev. Lett. 51, 39 (1983).

${ }^{31}$ M. Yameda, H.P. Furth, W. Heidbrink, A. Janos, S. Jardin, M. Okabayashi, E. Salberta, J. Sinnis, and F. Wysocki, in Proceedings of the Third Sympositum on Physics and Technology of Compact Toroids in the Mognetic Fusion Energy Program (Los Alamos National Laboratory, Los Alamos, New Mexico, 1980), p. 124 .

${ }^{32}$ A. Janos, S. Cowley, H. Hsuan, S. Paul, C. Skinner, F. Wysocki, and M. Yamada, in Proceedings of the Fourth Symposizm on the Physics and Technology of Compact Toroids (Lawrence Livermore National Laboratory, Livermore, California, 1981), p. 137.

${ }^{33}$ M. Yamada, R. Ellis Jr., H.P. Furth, G. Hart, A. Janos, S. Jardin, F. Levinton, D. Meyerhofer, M. Mimura, C.H. Nam, S. Paul, A. Sperduti, S. Von Goeler, F. Wysocki, and P. Young, in Proceedings of the Tenth International Conference on Plasma Physics and Controlled Nuclear Fuston Research (IAEA, London, 1984), p. 535.

${ }^{34}$ S. Paul, A. Janos, D. McNeill, M. Newhouse, and M. Yamada, in Proceedings of the Fifth Symposium on the Physics and Technology of Compact Toroids (Mathematical Scjences Northwest, Inc., Belleyue, Washington, 1982), p. 122.

${ }^{35}$ J. Howard, B.W. James, and W.I.B. Smith, J. Phys. D: Appl. Phys. 12. 1435 (1979).

${ }^{36}$ A. Janos, Princeton Plasma Physics Laboratory Report PPPL-2066 (1985): submitted to Phys. Fluids.

${ }^{37}$ T. Sato, T. Hayashi, and Y. Oda, in Proceedings of the Sixth U.S.

Symposium on Compact Toroid Research and the Fifth U.S.-Japan Joint 
Symposium on Compact Toroid Research (Princeton Plasma Physics Laboratory, Princetor, New Jersey, 1984), p. 33.

${ }^{38}$ The or ginal S-1 proposal included an active pinch c1.il, corresponding roughly to the position of the outer hoop, which was intended to aid formation. The outer hoop is, in essence, acting as a passive pinch coil. ${ }^{39}$ G.C. Goldenbaum, R.A. Hess, and R.S. Show, in Proceedings of the Seventh Compact Torus Symposium (Lcs Alamos Nationai Laböratory, Los Alamos, New Mexico, 1985).

'C.W. Barnes, H.W. Hoida, I. Henins, J.C. Fernández, T.R. Jarboe, and G.J. Marklin, in Proceedings of the Seventh Compact Torus Symposium (Los Alamos National Laboratory, Los Alamos, New Mexico, 1985). ${ }^{41}$ C.W. Barnes, T.R. Jarboe, I. Henins, A.R. Sherwood, S.O. Knox, R. Gribble, H.W. Hoida, P.L. Klingner, C.G. Lilliequist, R.K. Linford, D.A. Platts, R.L. Spencer, and M. Tuszewski, Nucl. Fusion 24, 267 (1984). ${ }^{42}$ E.O. Johnson and L. Malter, Phys. Rev. 80, 58 (1950). ${ }^{4}$ F.F. Chen, in Plasma Diagnostic Techniques, edited by R.H. Huddlestone and S.L. Leonard (Academic, New York, 1965), p. 178.

${ }^{44}$ S. Paul, F. Wysocki, and G. Hart, in Proceedings of the Sixth U.S. Symposium on Compact Toroid Research and the Fifth U.S.-Japan Joint Symposium on Compact Toroid Research (Princeton Plasma Physics Laboratory, Princeton. New Jersey, 1984), p. 109.

${ }^{45}$ J.B. Taylor, Phys. Rev. Lett. 33, 1139 (1974).

${ }^{46}$ I.G. Brown, A.B. Compher, and W.B. Kunkel, Phys. Fluids 14, 1377 (1971).

${ }^{47}$ E. Rebhan and A. Salat, Nucl. Fusion 18, 1431 (1978).

${ }^{48}$ H. Takahashi, K. Bol, H. Maeda, M. Okabayashi, and M. Revsch, Nucl. Fusion 22, 1597 (1982).

${ }^{49}$ C. Pfersich, R. Gruber, and F. Troyon, Nucl. Fision 23, 1127 (1983). 
${ }^{50}$ F.J. Wysncki and R.C. Grimm, Princeton Plasma Physies Laboratory

Report PPPL-2I62 (1984); accepted for publication in J. Comput. Phys (1985).

${ }^{51}$ M.N. Bussac, H.P. Furth, M. Okabayashi, M.N. Rosenbluth, and

A.M.M. Todd, in Proceedings of the Seventh International Confe "ence on

Plasma Phystics and Controlled Nuelear Fusion Research (IAEA, Innsbruck, 1978), p. 249.

\section{DISCLAIMER}

This report was prepared as an accounl of work sponsored by an agency of the United States Gowernment. Neitber the United States Government nor any agency thereor, nor any of their employees, makes any warranty, express or implied, or assumes any legal liability or responsibility for the ascuracy, completeness, or usefulness of any information, apparatus, product, of process disclosed, or represents that its use woukd not infringe privately owned rights. Reference hercin to any specific commercial produch, process, or service by trade name, trademark. manufacturer, or otherwise does not necessarily constitule or imply its endorsement, recommendation, or favoring by the United States Government or any agency thereof. The views and opinions of authors expressed herein do nol necessarily state or reflect those of the United Siates Goveinenent or any agency thereol. 


\section{TABLES}

\begin{tabular}{|l|l|r|}
\hline Wall Material & Thickness & Decay Time \\
\hline Stainless Steel & $0.51 \mathrm{~mm}$ & $12 \rightarrow 15 \mu \mathrm{s}$ \\
Stajnless Steel & $1.59 \mathrm{~mm}$ & $35 \rightarrow 45 \mu \mathrm{s}$ \\
Copper & $1.59 \mathrm{~mm}$ & $1.6 \rightarrow 2.0 \mathrm{~ms}$ \\
\hline
\end{tabular}

Table 1: Axisymmetric Resistive Decay Times for Solid Wall Stabilizers.

\begin{tabular}{|c|c|c|c|c|c|c|c|}
\hline $\begin{array}{c}\text { Time } \\
(\mu \mathrm{s})\end{array}$ & $\begin{array}{c}\theta \\
(\mathrm{deg} .)\end{array}$ & $\begin{array}{c}\overline{\delta B_{x}} \\
(\mathrm{G} / \mathrm{A})\end{array}$ & $\begin{array}{c}\overline{\delta B} \\
(\mathrm{G} / \mathrm{A})\end{array}$ & $\begin{array}{c}\overline{\delta B} \\
(\mathrm{G} / \mathrm{A})\end{array}$ & $\begin{array}{c}I_{0} \\
(\mathrm{kA})\end{array}$ & $\begin{array}{c}B_{0} \\
(\mathrm{G})\end{array}$ & $\begin{array}{c}B_{\text {edge }} \\
(\mathrm{G})\end{array}$ \\
\hline 56 & 1 & .0104 & 0 & .0104 & 44 & 333 & 401 \\
87 & 20 & .0108 & .0061 & .0131 & 30 & 306 & 309 \\
94 & 40 & .0122 & .0162 & .0258 & 24 & 284 & 219 \\
98 & 60 & .0147 & .0423 & .0859 & 21 & 271 & 117 \\
\hline
\end{tabular}

Table 2: Parameter Dependence on Time and/or Theta for Comparison with the Line-Tying Model. 


\section{FIGURTS}

FiG. 1. Diagram of Proto S-1/C showing the flux core, equilibrium "vertical" field coils, wall stabilizers, magnetic probe, and Thomson scattering layout.

FIG. 2. Contour plots of experimental poloidal flux, at $\theta=5^{\circ}$, for discharges with
(a) thin stainless steel walls
(b) thick stainless steel walls
(c) thick copper walls.

F1G. 3. Qualitative effect of plasma-wall boundary conditions and related equilibrium shape on tilting dynamics.

FIG. 4. Tilt angle (in degrees) versus time for the discharges in Fig. 2.

FIG. 5. Calculated induced currents in a spherical wall acting to stabilize the tilt mode. ${ }^{15}$

FIG. 6. Coniour plots of experimental poloidal flux, at $\theta=5^{\circ}$, for discharges with the "wagon wheel" stabilizer with 20 spokes and
(a) no connectors
(b) 10 connectors
(c) 20 connectors.

FIG. 7. Tilt angle (in degrees, plotted linearly) versus time for the case in Fig. 6 with 20 connectors.

FIG. 8. Detachment value versus magnetic axis major radius (at $\theta=5^{\circ}$ ).

FIG. 9. Growth rate versus magnetic axis major sadius.

FIG. 10. "Lifetime" vèrsus magnetic axis major radius.

FIG. 11. Position of the Rogowski coils an the "wagon wheel" stabilizer.

FIG. 12. Measured current pattern in the "wagon wheel" stabilizer (without connectors). On the left anc contour plots of constant $B_{t}$ (toroidal field) in 
steps of $150 \mathrm{G}$, on the right are the measured current patterns for $\mathrm{t}=56,80$, and $90 \mu \mathrm{s}$. (The length of the arrow indicates the magnitude of the current.) For the current patterns at $t=80$ and $90 \mu \mathrm{s}$, the toroidally averaged values have been subtracted out.

FIG. 13. Schematic diagram of the line-tying model.

(a) viewed parallel to the tilt axis.

(b) viewed perpendicular to the tilt axis.

FIG. 14. Predicted growth rate versus $\theta$

(a) assuming

$\gamma_{\theta}:$ no $\theta$ dependence in either $\alpha(=0.313), \xi(=32.9)$, or $\gamma_{0}(=0.766)$; and $7_{\alpha}$ : including only the experimentally measured $\theta$ dependence in $\bar{\delta} \ddot{B}$, which enters in $a$.

(b) for $A=340 \mathrm{~cm}^{2}, L=11.7 \mathrm{~cm}$ and $A=200 \mathrm{~cm}^{2}, L=23.4 \mathrm{~cm}$, including all known $\theta$ dependencies. The nivasurcd growth rate is also indicated.

FIG. 15. Predicted growth rate and magnitude of the perturbed line-tying current versus time.

(a) Predicted tilt angle (in degrees) for the same conditions in Fig. 14(b). The $(\bullet)$ indicates the measured data.

(b) Predicted magnitude of the perturbed line-tying current for the same conditions in Fig. 14(h). The $(\bullet)$ indicates the measured data. (note: the predicted $I_{\text {l.t. }}$ values for the case $A=340 \mathrm{~cm}^{2}, L=11.7 \mathrm{~cm}$ have been reduced by the factor $200 / 340$ ).

F]G. 16. Experimental scaling of $T_{e}$ ad.d $n_{\epsilon}$ with toroidal plasma current.

FIG. 17. Scaling of $n_{e} \gamma$ with plasma c1.rrent.

(a) Experimental scaling of $n_{e} \gamma$ with toroidal plasma current for discharges with 0.51-mm stainless stee] wall and 10 spoked "wagon wheel" stabilizers. The discharges had $17.5-\mathrm{cm}$ magnetic axis major radii. 
(b) Experimental data comparing 10 and 20 spoked "wagon wheel" stabilizers. The discharges had $15.5-\mathrm{cm}$ magnetic axis major radii.

FIG: 18. Experimental scaling of $n_{e} \gamma$ with toroidal plasma current for discharges with the "full enc.sosure" stabilizer (20 connectors). Included are the results from discharges with both $12-\mathrm{cm}$ and $16-\mathrm{cm}$ magnetic axis major radji. 
\# $85 \times 2016$

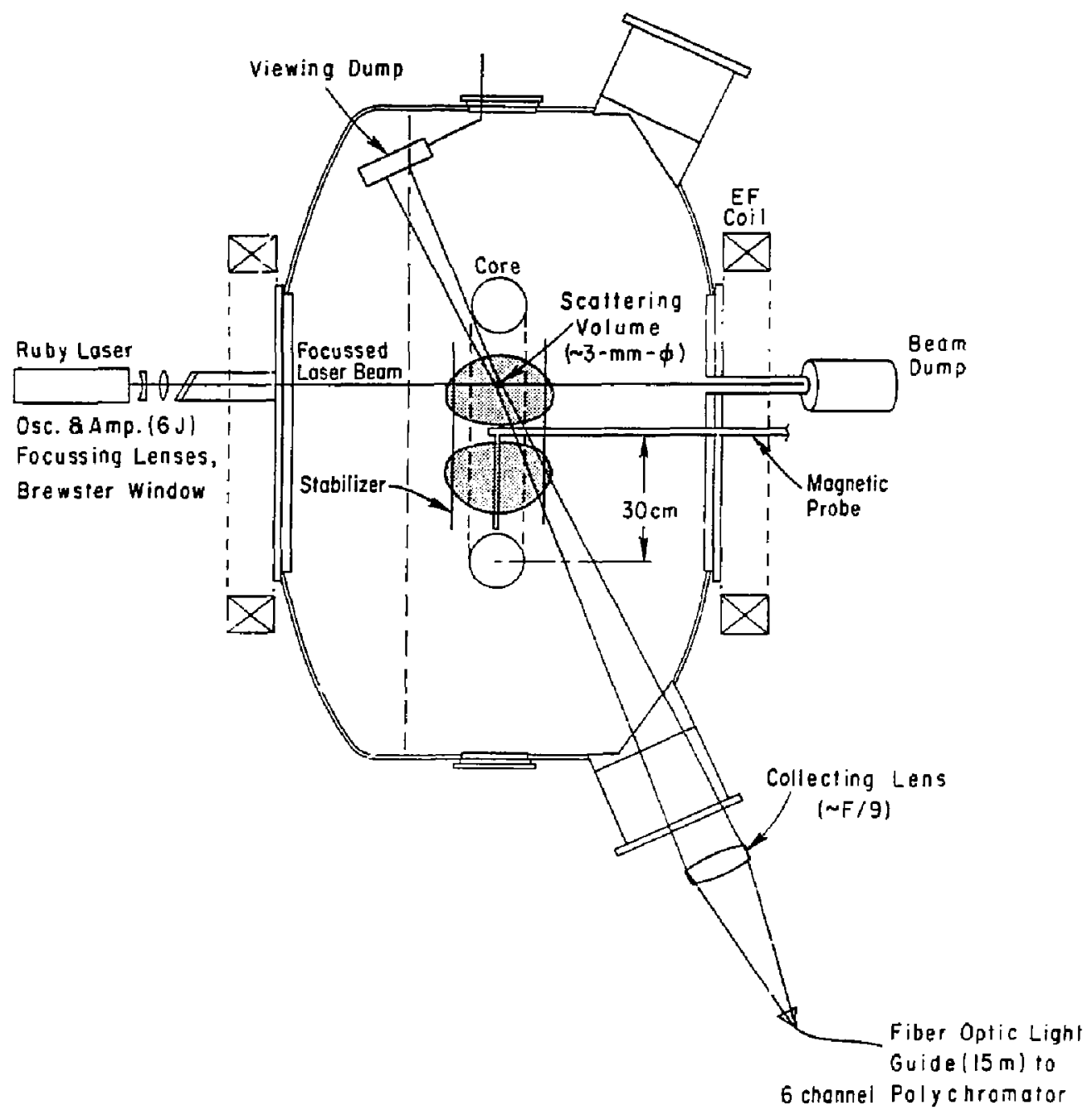

Fig. I 


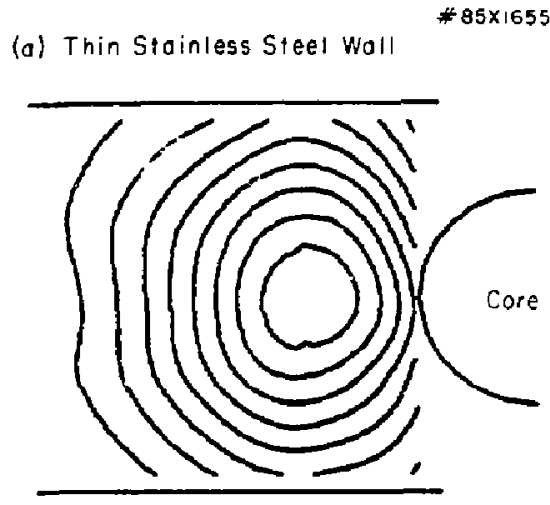

(b) Thick Stainless Steel Wall

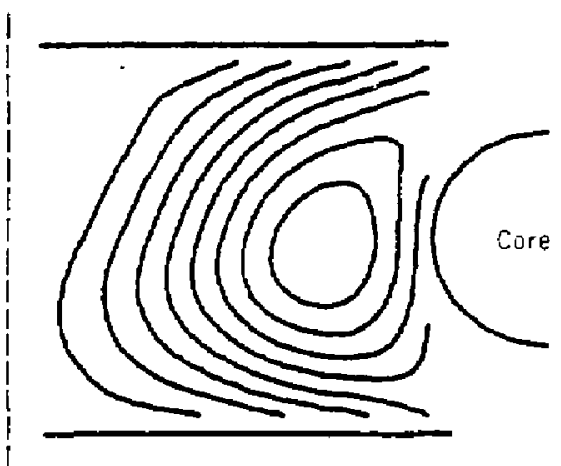

(c) Thick Copper Wall

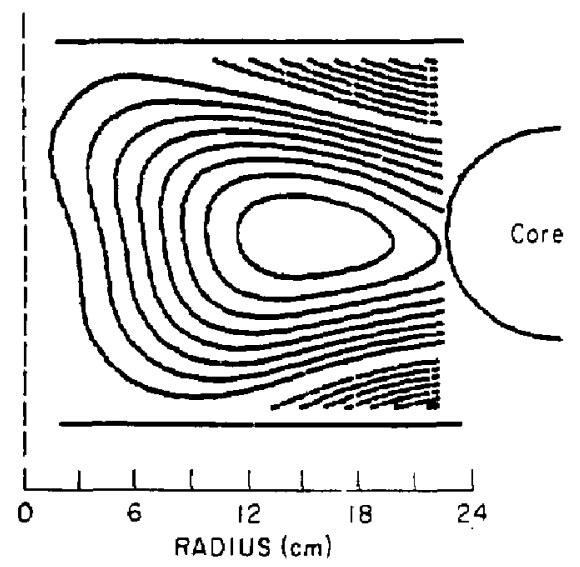

Fig. 2 
\# $84 \times 0226$

Open Field Lines

"Line Tied" To a Horizontal Wo!l
Outer Closed Field Lines

"Line Tied" To a Horizontal Wall

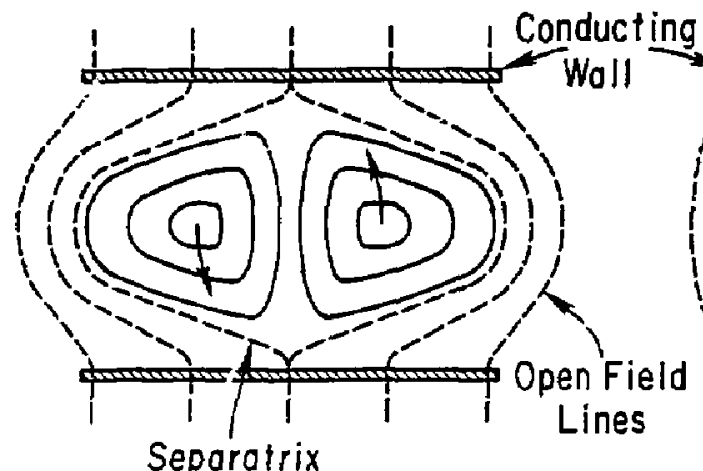

Separotrix
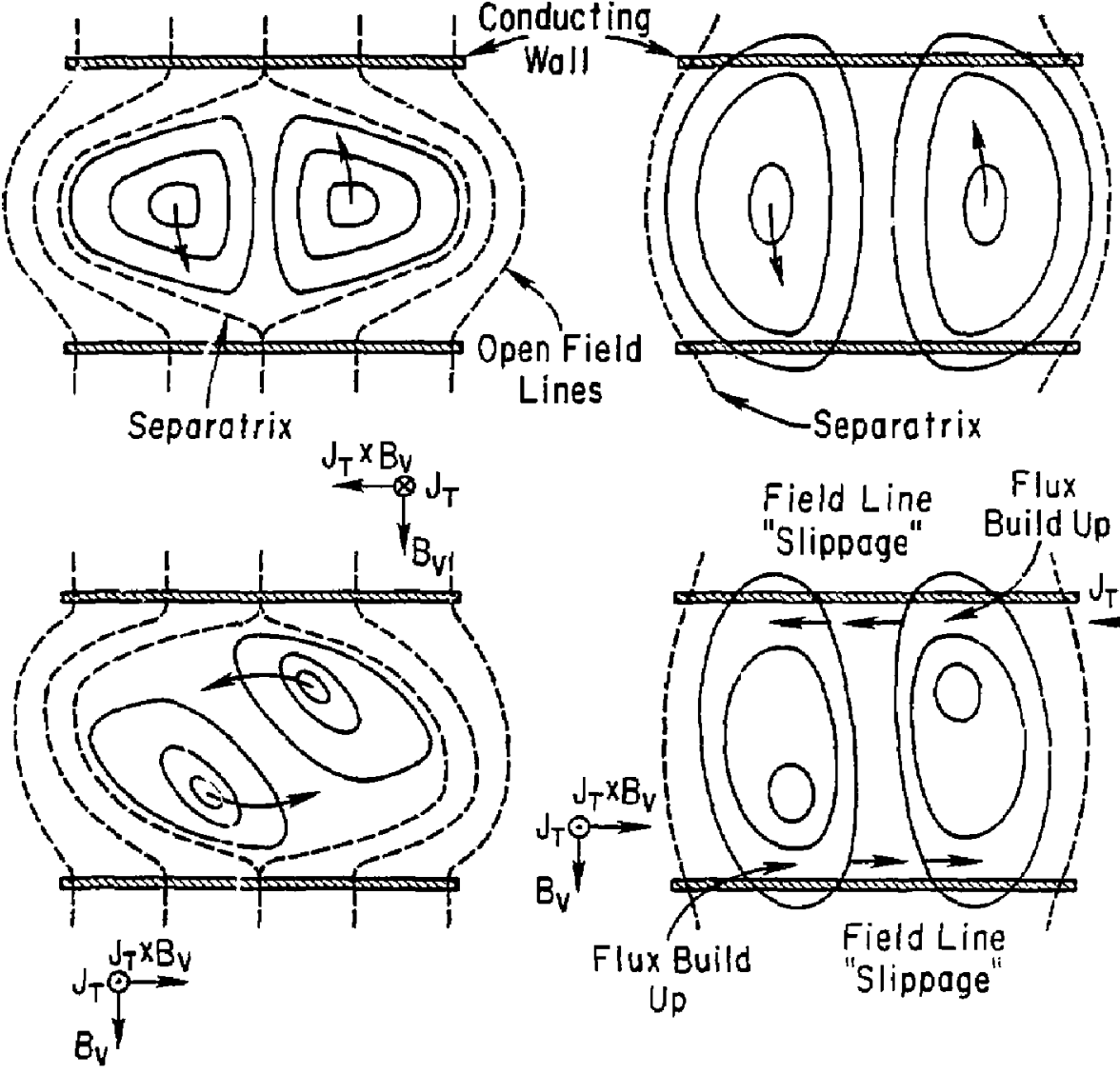

Flux Build

Up

Field Line Flux

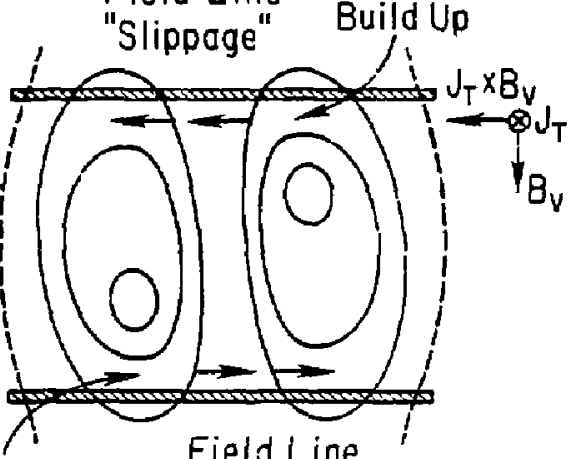

Field Line

"Slippage" 


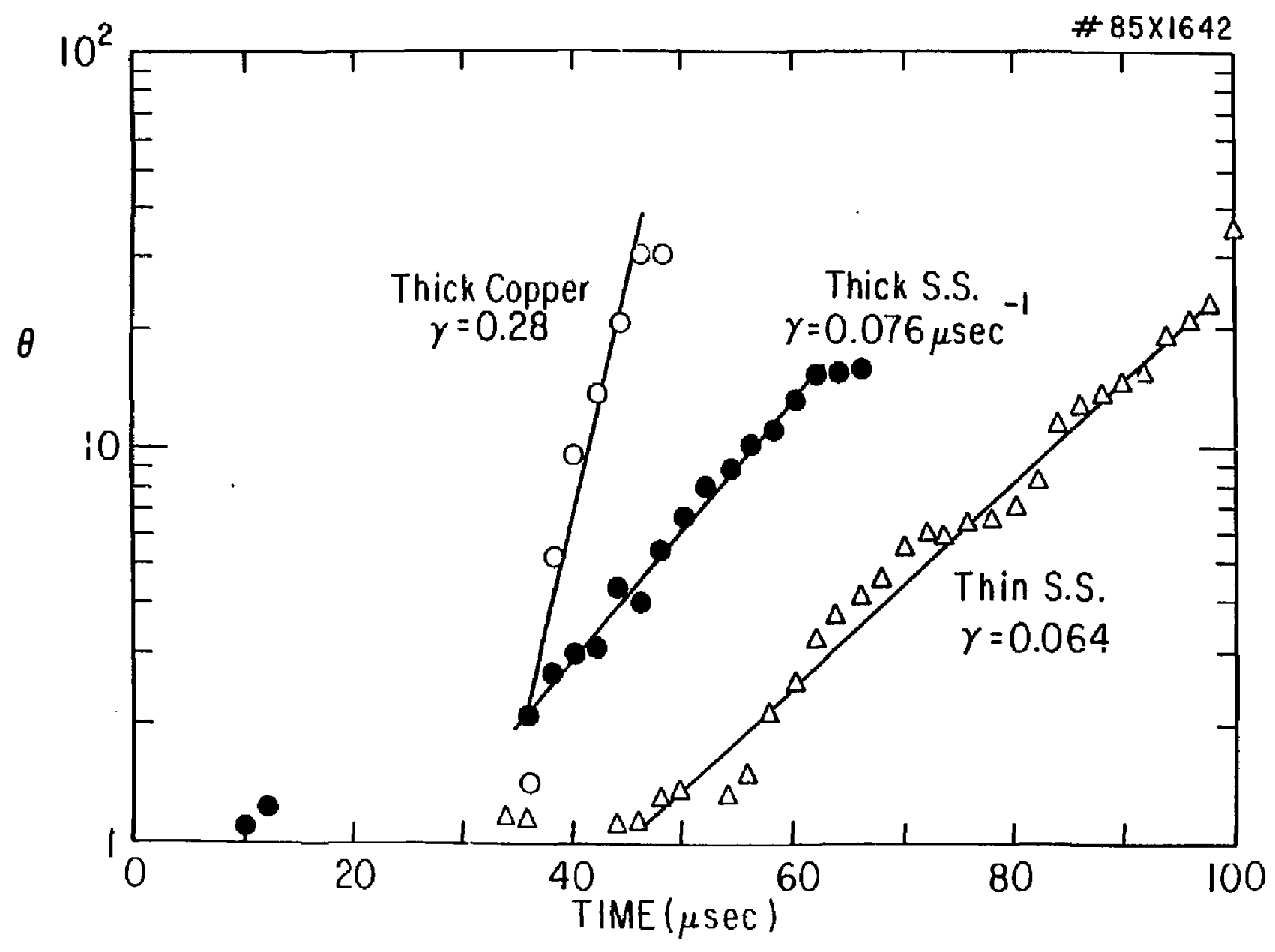


8
8
$\frac{8}{0}$
\#
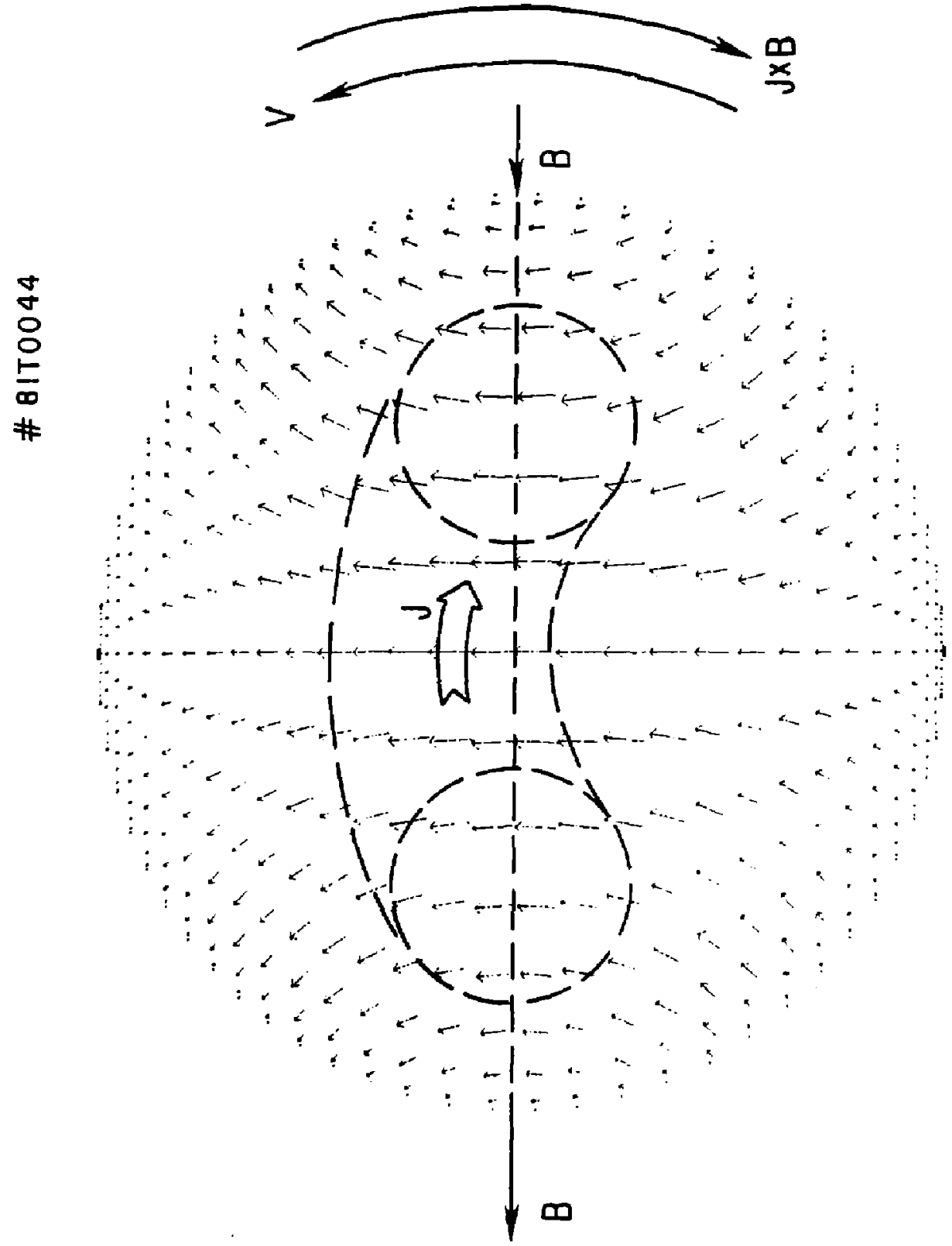
(a) Wogon Wheels With 20 Spokes

$\# 85 \times 1654$

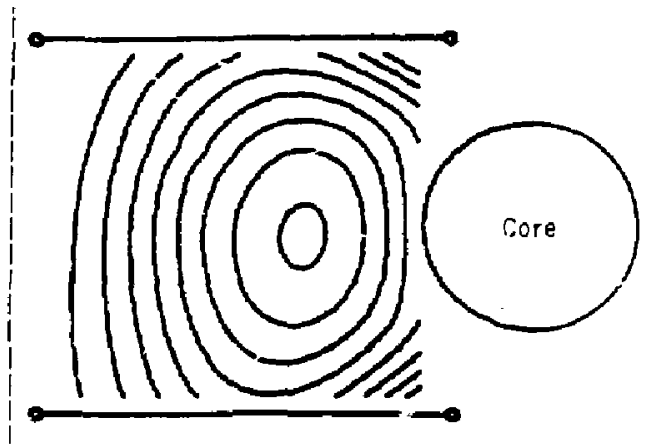

(b) Full Enclasure With 10 Connectors

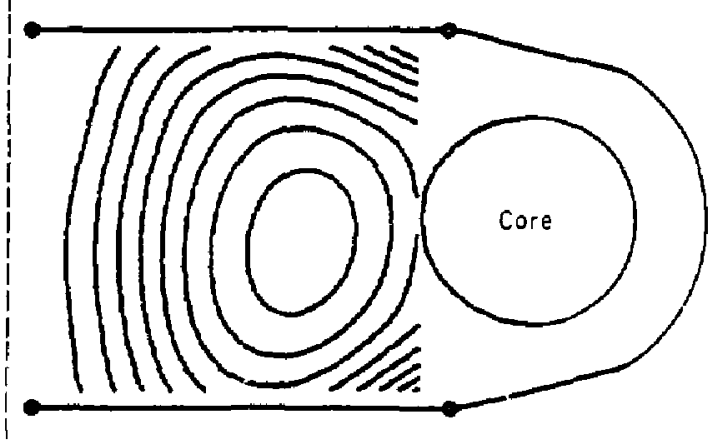

(c) Full Enclosure With 20 Connectors

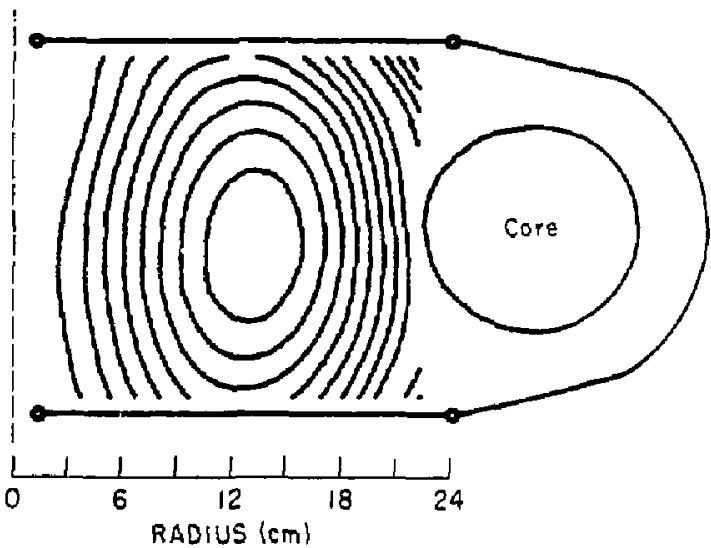

Fig. 6 


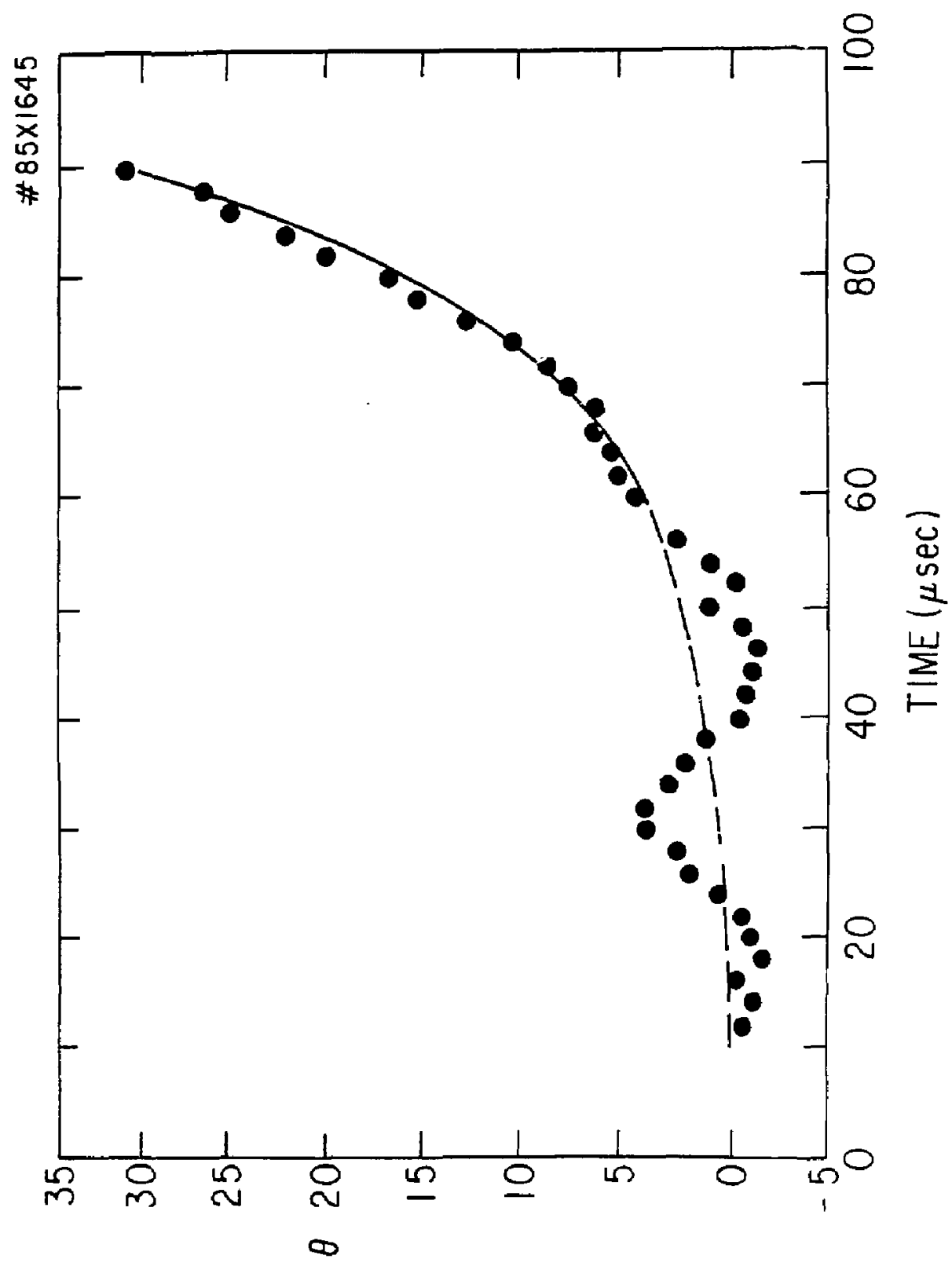




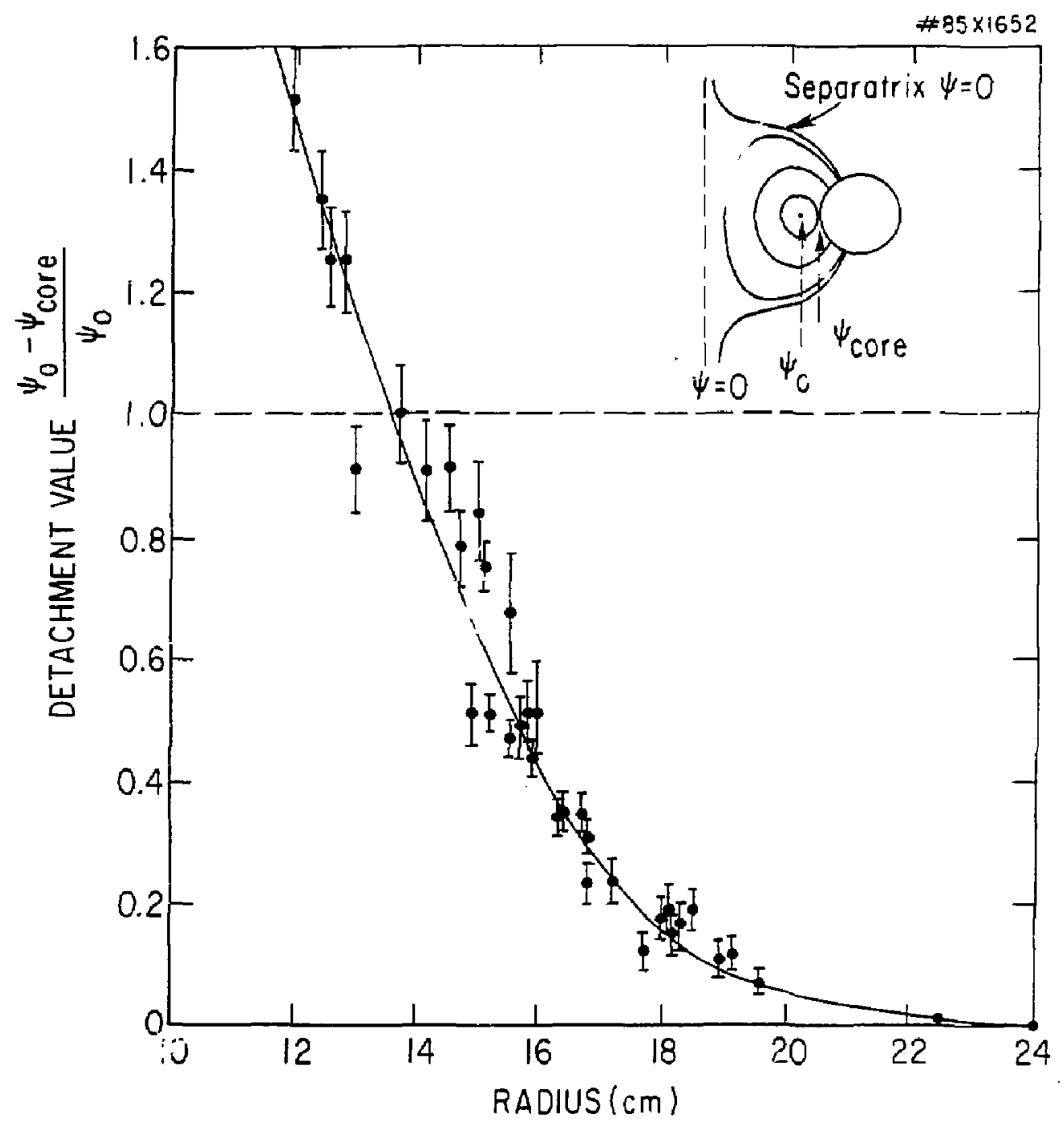

Eig. 8 


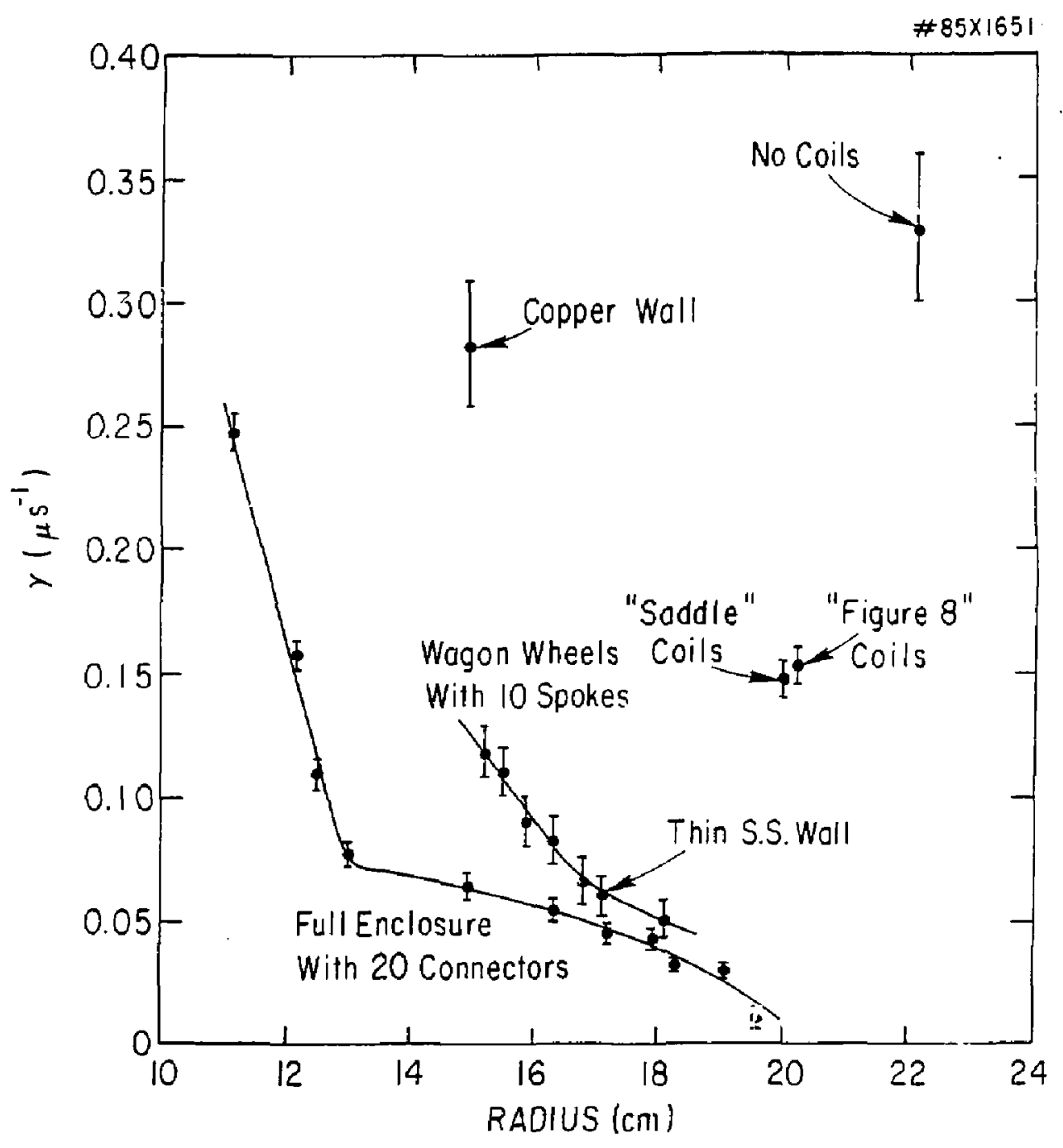

Fig. 9 


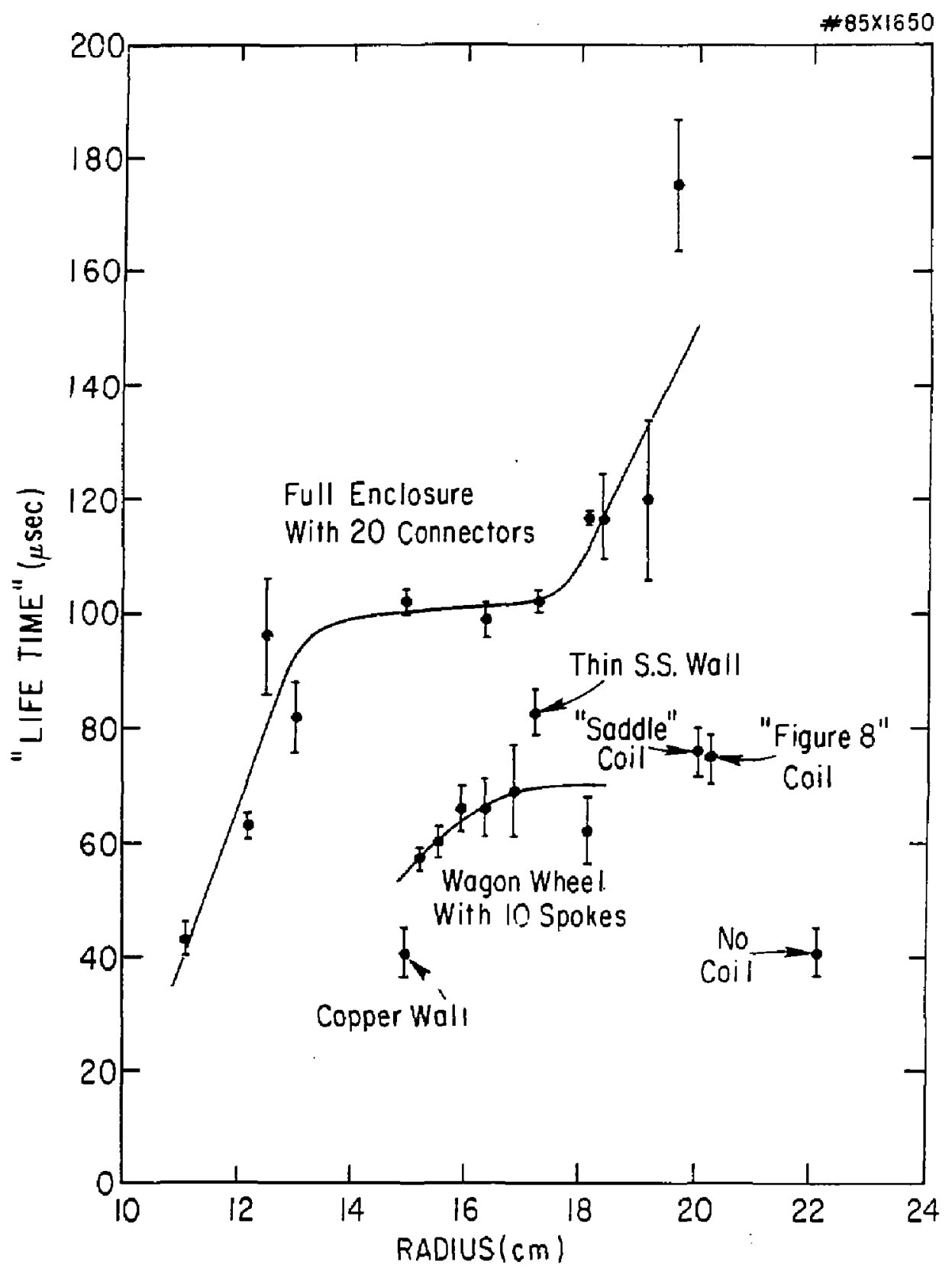

Fig. 10 


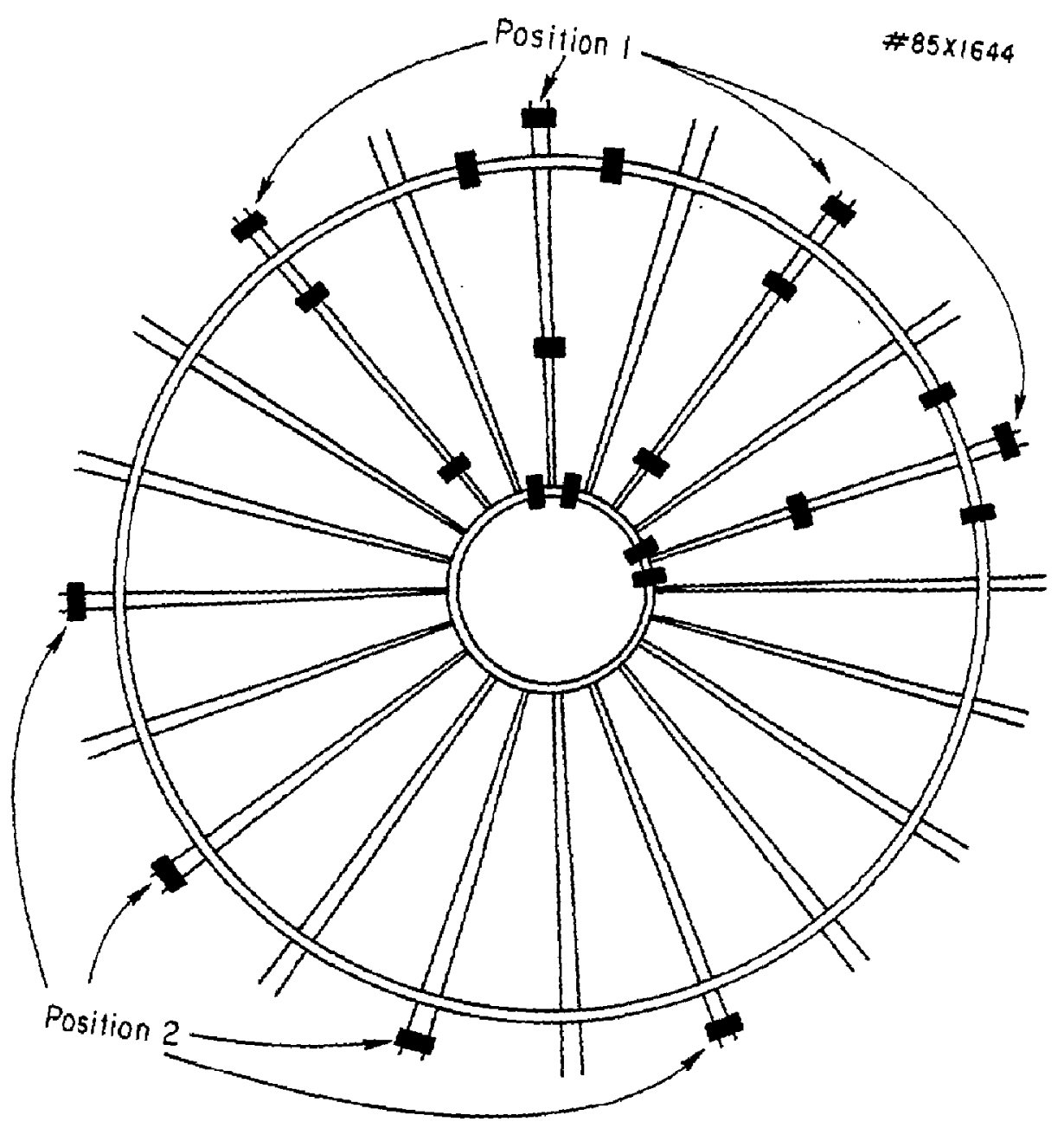

Eig. 11 

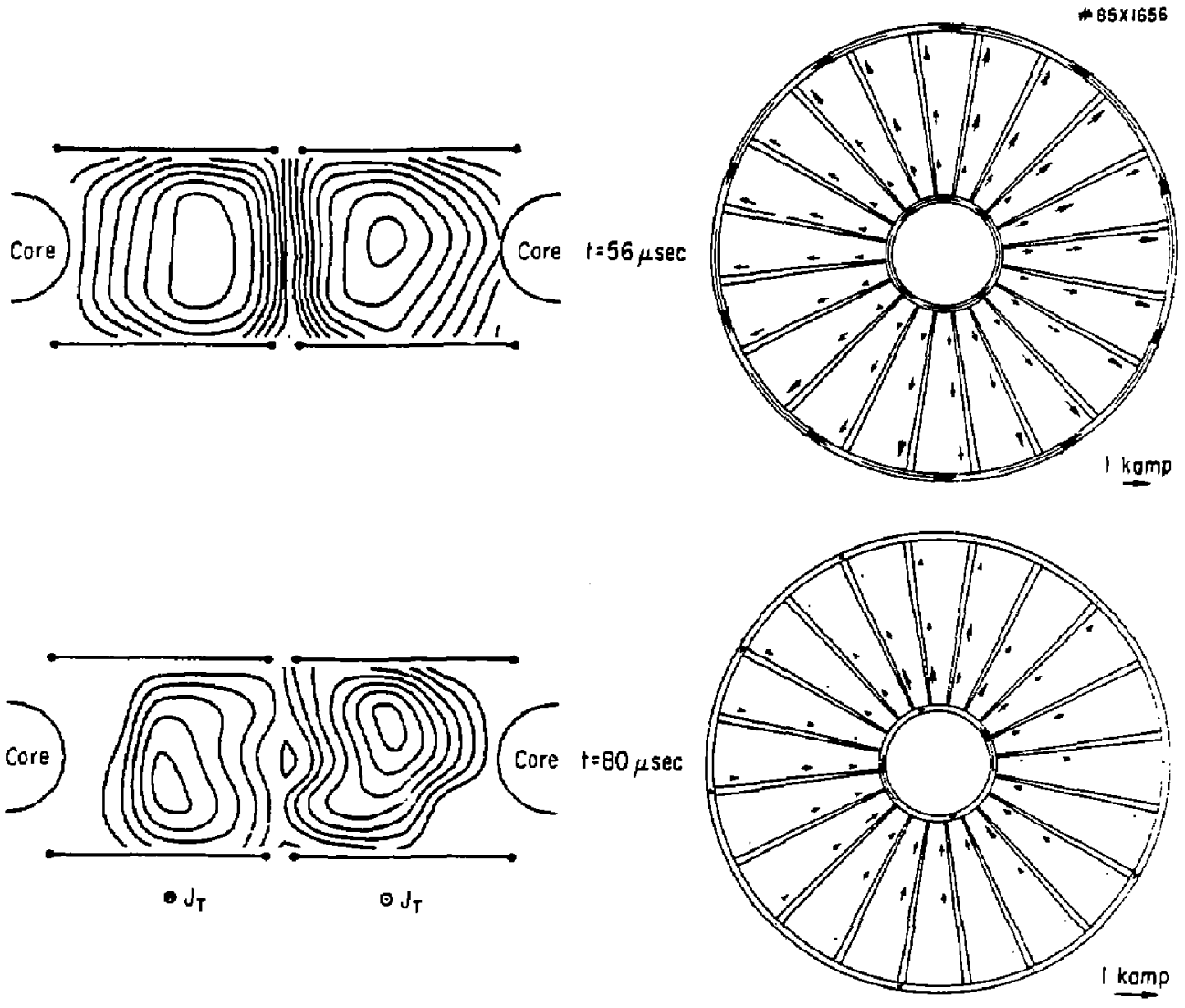

\section{- Tilt Axis}
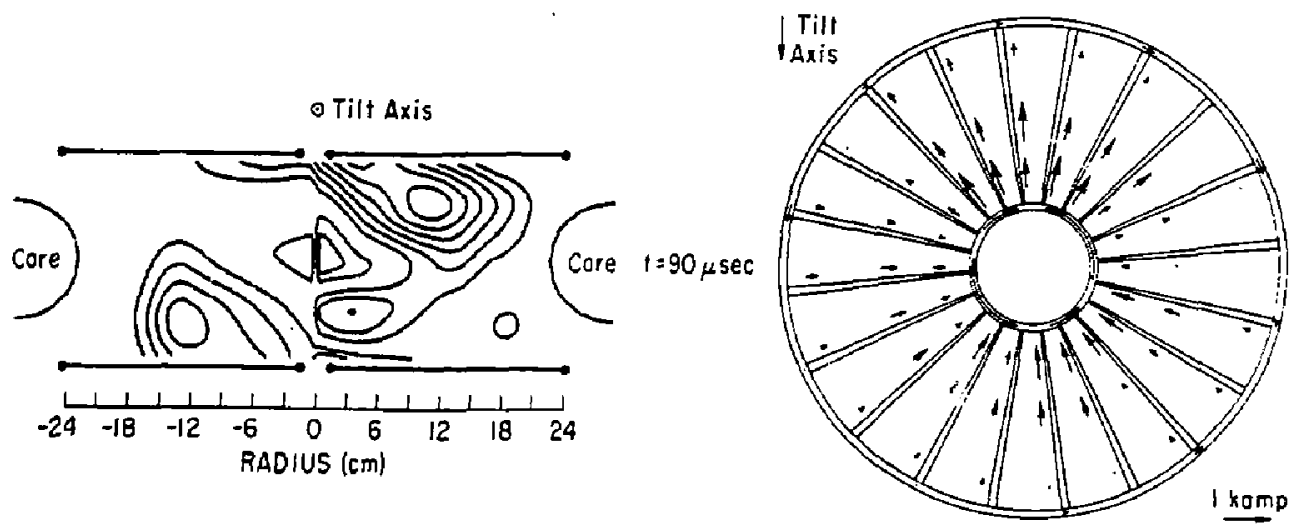

Fig. 12 
$\# 85 \times 1643$

(a)

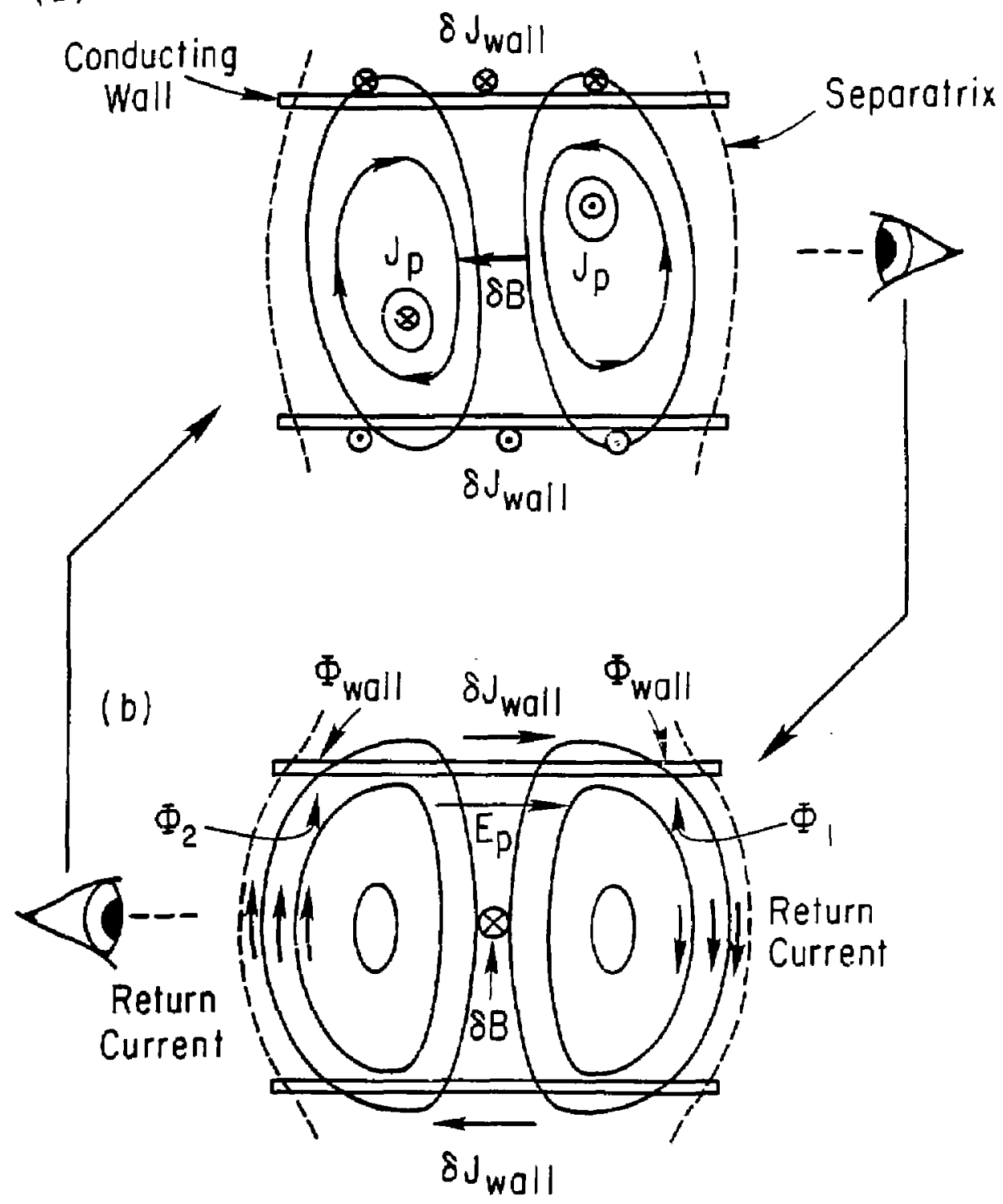

Fig. 13 

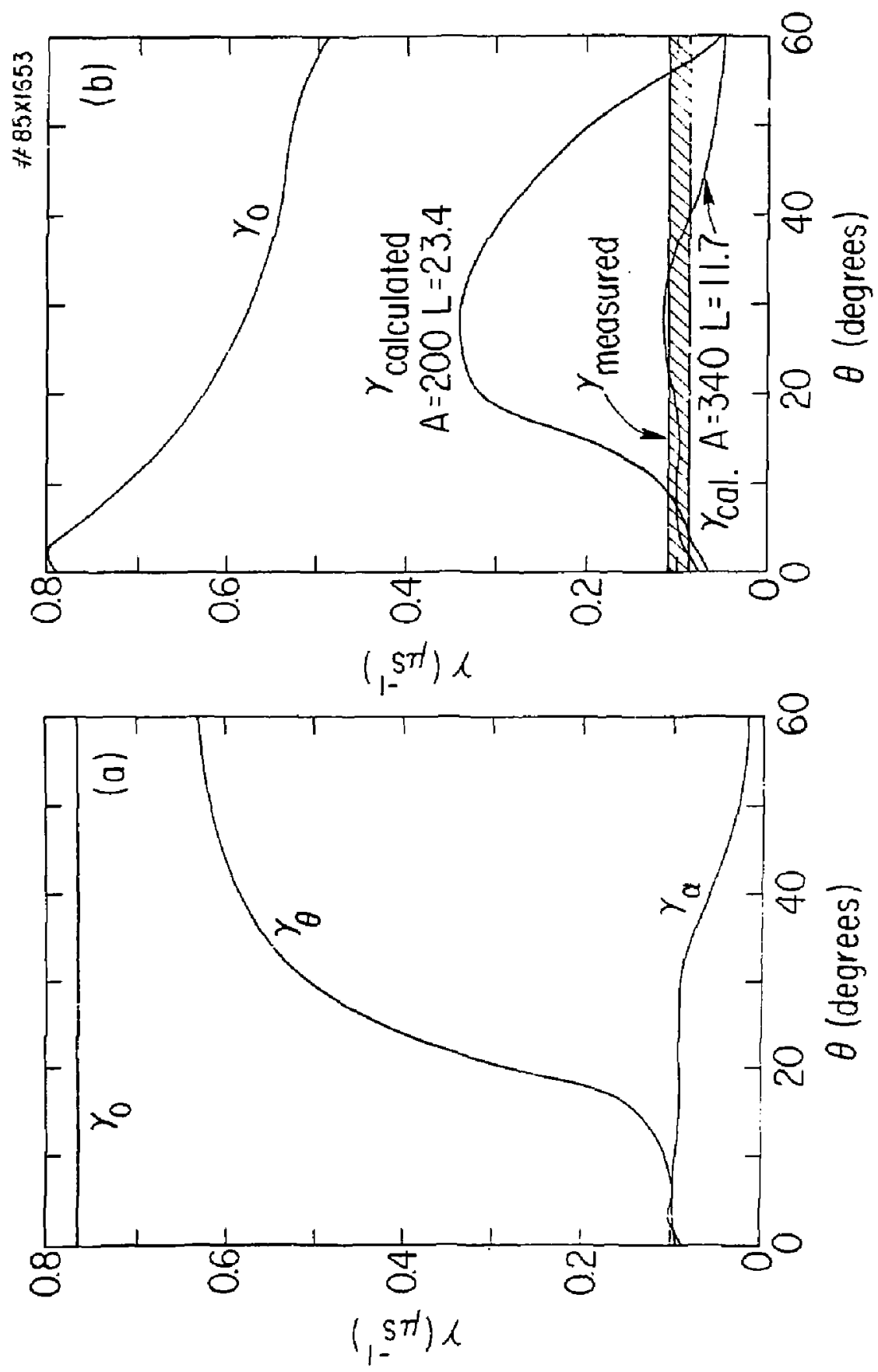

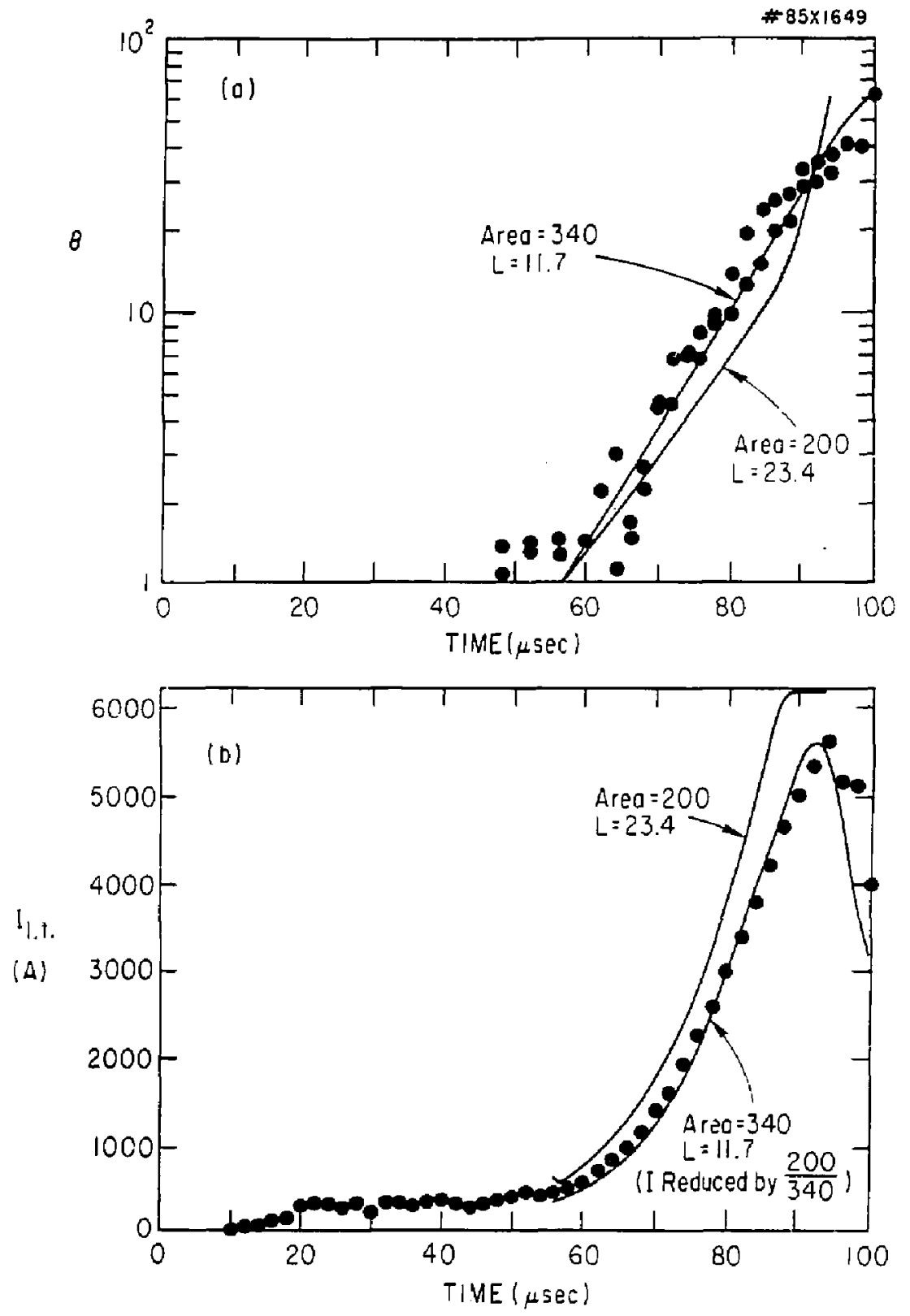

F士g. 15 


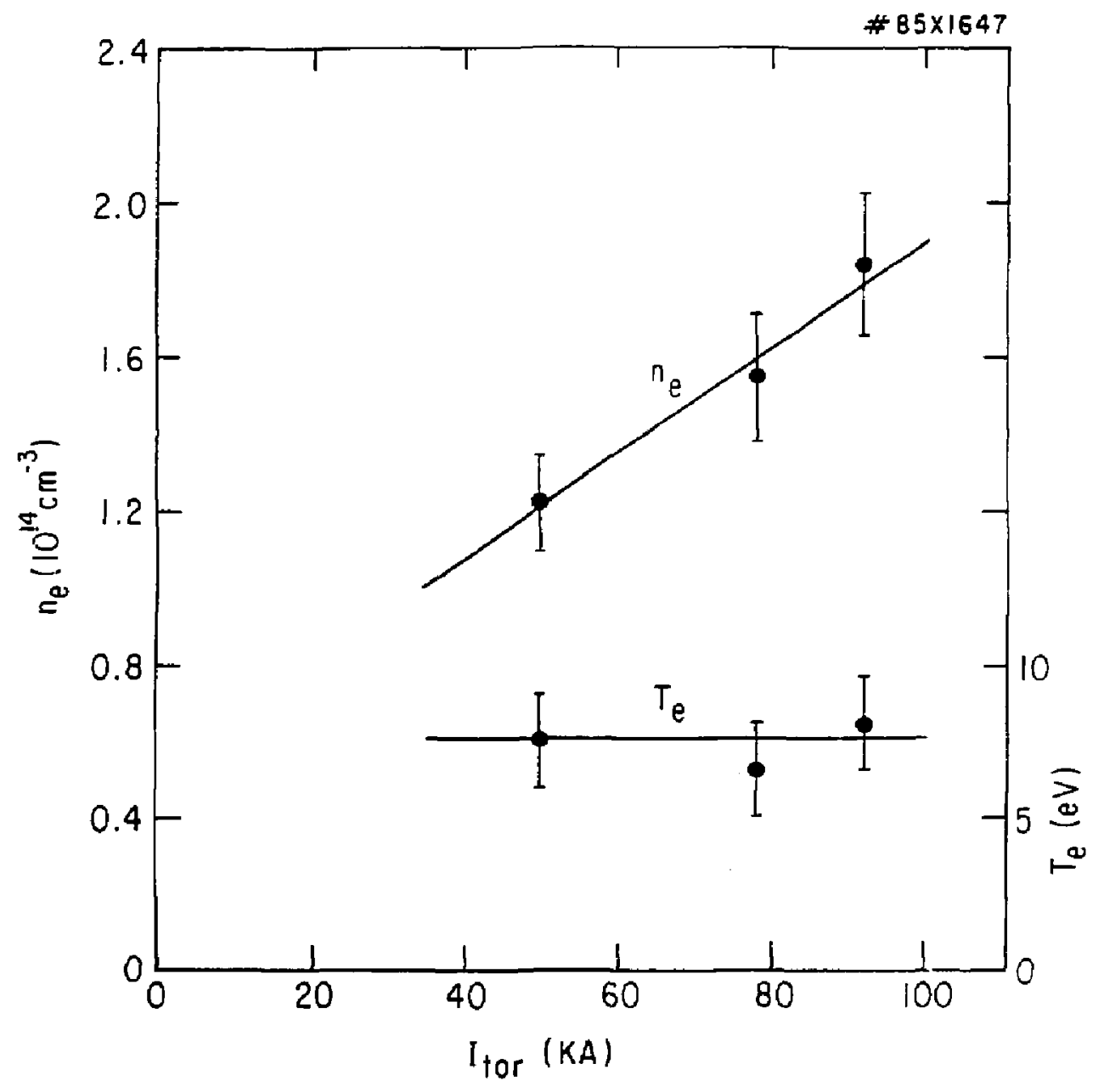

Fig. 16 

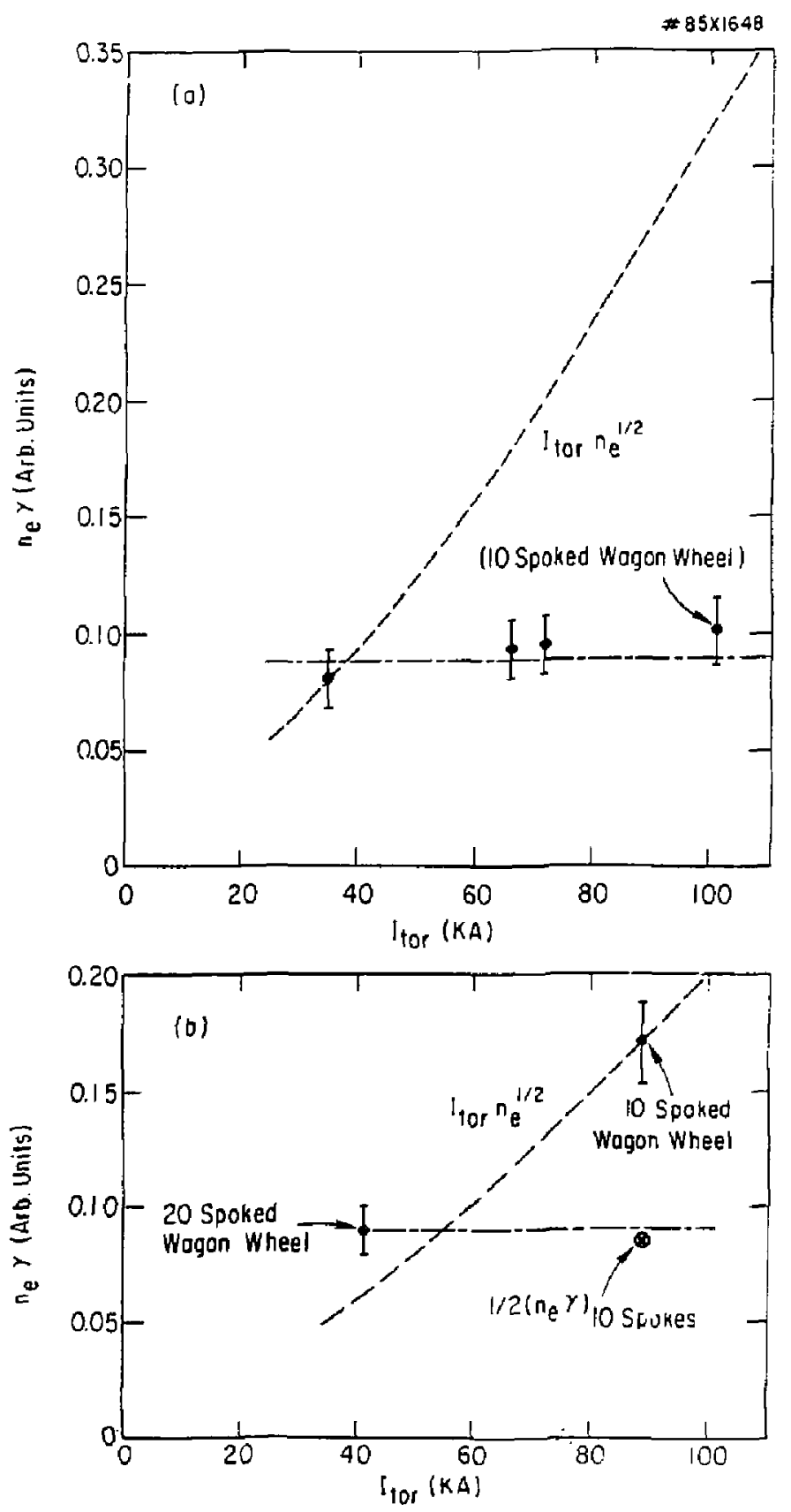

Eig. 17 


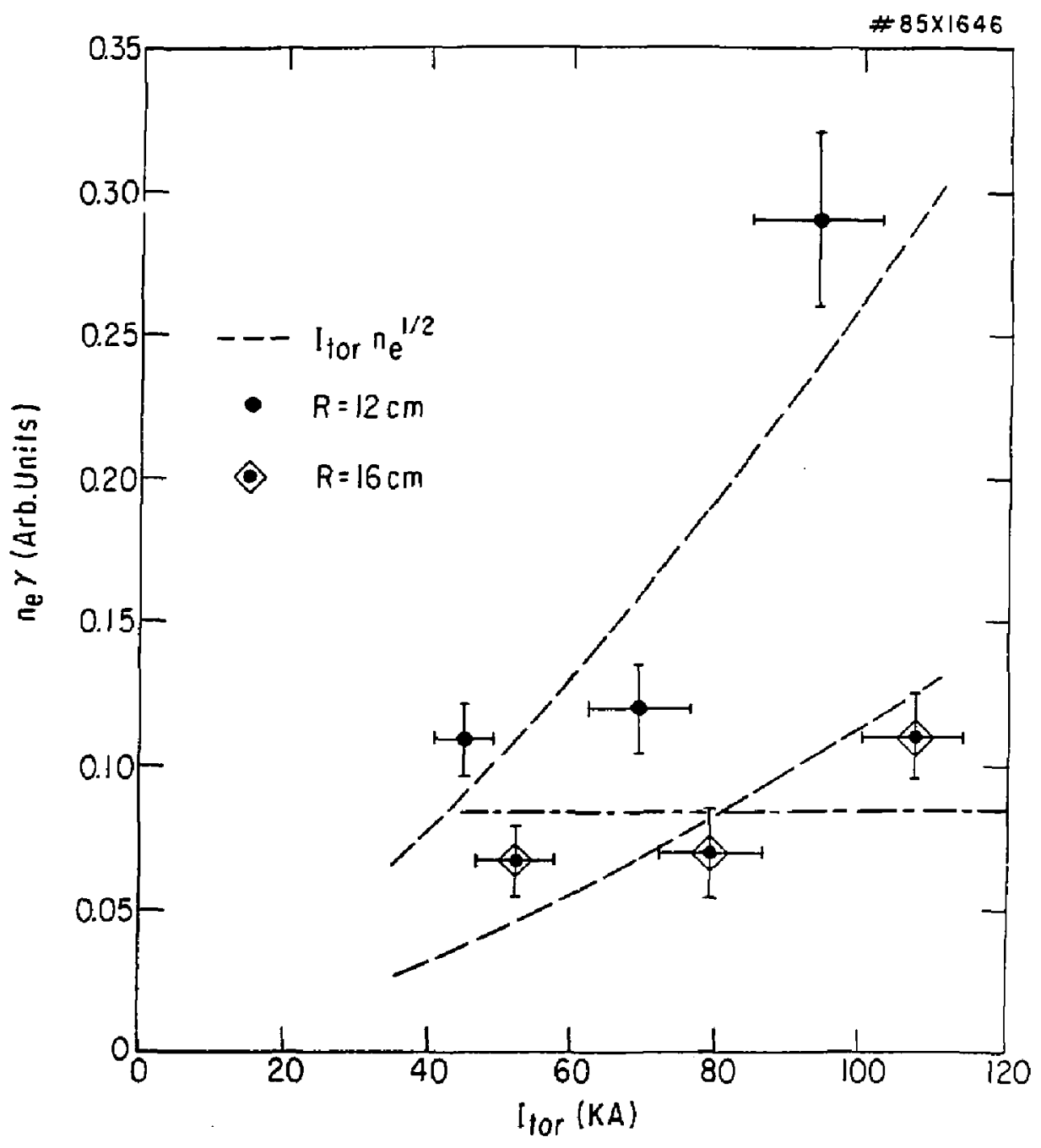

Fig. 18 


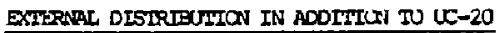

Plasta Res Lab, Austar Atac'l Univ, AUSTRALIA

T. Frank J. Paoloni, Luiv of wollongang, ALSIBAtIA

Prot. I.R, Jones, Flinders Univ,, AUSLPALIA

Prof. M.H. Exennan, Uhiy Sydney, AustraLIA

PtoE. F. Cav, Inst Theo PIys, ALSTRIA

Ptof. Erark vertest, Inst theoretische, BsugIuM

Dr. D. Ralunbo, Dg XII fluion Prog, BEIGIUH

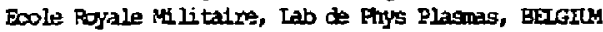

DE. P.H. Sakanaka, Univ Estanial, ERAzI

DI. C.R. James, Univ of Alberta, CAropa

Frof. J. Teictmann, Liniv of Mcontreal, Caspon

T. H.H. Skarsgard, Univ of Saskatchewan, CANoin

Prot. 5.R. 5reenivasan, University of Calgary, CANBCA

Prof. Tubor \%. Johnston, IRS-Energie, CARDA

Dr. Harmes Bqtard, Univ Eritisin Colunibia, Canad

Dc. i1.P. Badrynski, HQB Tednologies, Inc., CANAzi

Calk River, Nucl Lab, CarapA

Znengur is, sin Inst Fyysies, Crma

ilibrary, thing the thiversity, CrIt

Iibxarian, Institute of Fhysics, CHINA

Inst Plasira Fys, Apaderila Sinica, GmA

D. Beter Lukac, Komenskeho Univ, CzectosLONAKTA

The Librarian, Oulham Labocabory, ENGLAND

Prof. schatzman, Obsertatoire de vice, FRANCE

J. Radet, COH-EP6, FRANCE

AN Dupas libary, aM Dupas Iibcary, ERANCE

Dr. Tho Mal, Acadeny Bibliographic, HOG kONG

Preguint Library, Cent Res Inst Phys, Huxtary

DT. R.K. Chijajlani, Vikran thiv. IUDA

DT. B. Laggupta, Saha Inst, INDIA

Dr. D. Kaw, Frysical Research Lab, DDFA

Dr. Thillip fosenay, Inrael Inst Mech, ISRAEI

Proxi. 5. Oyperman, Del Aviv thiversity, ISRAEL

Prof. G. Rostagni, Univ DI Padowe, IIALY

ilibcarsn, Int'l Ctr Theo Phys, IILLY

iss Clelia De Palo, Agsoc DURATOM-2NEA, IIALY

Biblioteca, tel OR ERATY, IRLY

Dr. H. Yarato, nosidiba Fes \& Dev, JAPAN

Direc. Dept. Ig. Twkanak Dev. JABRI, JAPAN

Prof. Abuyuki Inowe, thiversity of Tokyo, IAPAN

Research Info Center, Lagoya thiversity, JAPAN

Prof. Kyojt idshikawa, thiv of tiroshima, JupAN

Prof. Sigena Moti, JAERI, JAPAN

Erof. 5. Tanaka, Kyoto thiversity, APAN

Lilurary, kyot Univergity, JAPA

ProE. Ichilso kawakmi, whon thiv, IRPAN

Prof. Satoahi Itoh, Kyushu thiversity, JAPAy

Dr. D.I. Chai, Acv. Inst Bai \& Tech, KCREA

Iech Info Diviation, KAERI, IOREA

Bibliotheok, Fon-Ingt voor Plasna, NEIHLRLANDS

zrof. H.S. isley, University of wikato, Nei zFatand

Frof. J.A.C. Caber1, Inst Superlor Tecr, EORTueal
Or. Ortavian Betrus, AL,I uWA thiversity, fOMANIA Erof. M.A. Hellberg, thitersity of Natal, $D$ Aftich

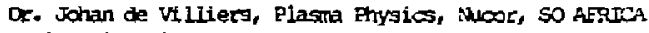
Fusion Div. Tibrary, JWN, SPAIN

Eroc. Hare wilheluson, chalmers univ Tech, sweul

De. Lemart 5tenflo, Chiversity of IMEA, SNEDS

Iibrary, Royal Inst Tech, SwBDaN

Centre de Recherchesen, Bcole Rolytecin Fed, SITLESLAw

DE. V.T. Tolok, Khadkov Fyys Iech Ins, ISSR

DE. D.D. Ryutov, Siberian Acad 5Ct, LSSR

DE. G.A. Eliseev, Murciatov Institate, ISSR

Dr. W.A. Glukhikh, Inst Electro-fhysical, LSSR

Institube Gen. Fysics, ISSR

Prof. T.J.M Boyd, Univ College N wales, WALES

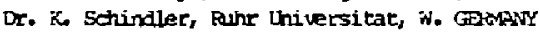

Nuclear Res Estab, Julich ted, H. GoraNI

Tibrarian, Mex-Plandk Instibut, H. GotaAY Bibliothek, Inst Plasmiforsctung, W. GOMANY Erof. R.K. Jorev, Injt Fhys, ruoustavia 